\title{
Schlicht regions for entire and meromorphic functions
}

\author{
M. Bonk* and A. Eremenko ${ }^{\dagger}$
}

\begin{abstract}
Let $f: \mathbf{C} \rightarrow \overline{\mathbf{C}}$ be a meromorphic function. We study the size of the maximal disc in $\overline{\mathbf{C}}$ with respect to spherical metric, in which a singlevalued branch of $f^{-1}$ exists. The problem is related to normality and type criteria. Best possible lower estimates of the size of such discs are obtained for entire functions and a class of meromorphic functions containing all elliptic functions. An estimate for the class of rational functions is also given which is best possible for rational functions of degree 7 . We do not know whether it is asymptotically best possible when the degree tends to infinity. For algebraic functions of given genus we obtain an estimate which is precise for genera 2 and 5 and asymptotically best possible when the genus tends to infinity.
\end{abstract}

\section{Introduction}

We consider the Riemann sphere $\overline{\mathbf{C}}$ equipped with the Riemannian metric coming from the length element $2|d w| /\left(1+|w|^{2}\right)$. Then the diameter of $\overline{\mathbf{C}}$ with respect to this metric is equal to $\pi$. The Riemann sphere can be isometrically embedded into $\mathbf{R}^{3}$ as the unit sphere. The distance between two points can be visualized as the angle between directions to these points from the center of the sphere. The Euclidean distance of two points $x$ and $y$ will be denoted by $|x-y|$ and spherical distance by $\operatorname{dist}(x, y)$. Open Euclidean discs will be $D$ or $D(a, r)$, and open spherical discs $B$ or $B(a, r)$, where $a$ is the center of the disc and $r$ is its radius. So $B(a, \pi / 2)$ is an open hemisphere.

Let $X$ be a Riemann surface, and $f: X \rightarrow \overline{\mathbf{C}}$ a non-constant holomorphic map. If $p \in X$ is not a critical point, there exists a holomorphic germ $\phi_{p}$

${ }^{*}$ supported by a Heisenberg fellowship of the DFG

${ }^{\dagger}$ partially supported by NSF grant DMS-950036 and by the Lady Davis Foundation 
of the inverse $f^{-1}$ at $w=f(p)$ such that $\phi_{p}(w)=p$. Let $d_{f}(p)$ be the spherical radius of the largest open spherical disc centered at $w$ to which $\phi_{p}$ can be analytically continued. Such a largest disc always exists. If $p$ is a critical point let $d_{f}(p)=0$. The spherical Bloch constant of the function $f$ is now defined as $\mathfrak{B}_{f}=\sup _{p \in X} d_{f}(p)$. For a class $\mathcal{M}$ of holomorphic maps we define the spherical Bloch constant as

$$
\mathfrak{B}_{\mathcal{M}}:=\inf _{f \in \mathcal{M}} \mathfrak{B}_{f}
$$

Our first result implies that for the class $\mathcal{E}$ of all non-constant entire functions $\mathfrak{B}_{\mathcal{E}} \geq \pi / 2$.

Theorem 1.1 Let $f \neq$ const be an entire function. Then for every $\epsilon>0$ there exists a spherical disc $B$ of spherical radius at least $\pi / 2-\epsilon$ and $\operatorname{dist}(B, \infty)<\epsilon$, such that a single-valued branch of $f^{-1}$ is defined in $B$.

On the other hand, the examples $f(z)=z^{2}$ and $f(z)=e^{z}$ show that $\mathfrak{B}_{\mathcal{E}} \leq \pi / 2$, so $\mathfrak{B}_{\mathcal{E}}=\pi / 2$. The following result shows that in fact Theorem 1.1 is sharp in a stronger sense.

Example 1.2 There exists a transcendental entire function $f$ such that no single-valued branch of $f^{-1}$ is defined in any open hemisphere.

It is known $[19,16]$ that for the set $\mathcal{L U}$ of locally univalent meromorphic functions in $\mathbf{C}$ the equality $\mathfrak{B}_{\mathcal{L U}}=\pi / 2$ holds. We give a more precise statement which implies this.

Theorem 1.3 Let $f$ be a locally univalent meromorphic function in $\mathbf{C}$. Then for every $\epsilon>0$ there exists an arc $l$ of a great circle which has spherical length at most $\pi$ such that a branch of the inverse $f^{-1}$ is defined in $\overline{\mathbf{C}} \backslash l_{\epsilon}$ where $l_{\epsilon}$ is the spherical $\epsilon$-neighborhood of $l$.

Again the exponential function shows that $\pi$ is best possible in this theorem, and similarly as in Example 1.2 one can construct an example showing that we cannot take $\epsilon=0$.

Theorems 1.1 and 1.3 can be viewed as type criteria. We recall the statement of the problem of type. Let $X$ be an open simply connected abstract surface and

$$
f: X \rightarrow \overline{\mathbf{C}}
$$

a topologically holomorphic map ${ }^{1}$. This means that every point $p \in X$ has a neighborhood $V$ and a local coordinate $z: V \rightarrow \mathbf{C}, z(p)=0$, and a local

\footnotetext{
${ }^{1}$ a. k. a. inner map in the sense of S. Stoïlov
} 
coordinate $w: f(V) \rightarrow \mathbf{C}$, such that $w \circ f=z^{m}$ in $V$ for some natural integer $m=\mu_{f}(p)$, which is called the local degree of $f$ at $p$. Given such a map $f$ there exists a unique conformal structure on $X$ which makes $f$ holomorphic. The problem of type is to determine from geometric properties of $f$, whether $X$ equipped with this structure is conformally equivalent to the complex plane (parabolic case) or to the unit disc (hyperbolic case). By geometric properties we mean those which are invariant under pre-composition of $f$ with any homeomorphism of $X$. To reformulate Theorem 1.1 as a type criterion we consider the following property of a function $f$ :

$\left(\mathbf{P}_{\epsilon}\right)$ every open disc $B$ in which a single-valued branch of $f^{-1}$ is defined has either spherical radius at most $\pi / 2-\epsilon$ or $\operatorname{dist}(B, \infty) \geq \epsilon$.

Then we have

Theorem 1.4 If $f$ in (1) omits $\infty$ and satisfies $\left(\mathbf{P}_{\epsilon}\right)$ with some $\epsilon \in$ $(0, \pi / 2)$, then $X$ is hyperbolic.

Theorem 1.3 can be similarly reformulated.

The spherical Bloch constant for the whole class of non-constant meromorphic functions in the plane is not known. Ahlfors [1] obtained the lower estimate $45^{\circ}$ as a corollary from his celebrated Five Islands Theorem. The best known lower estimate $60^{\circ}$ is due to Minda [16]. The conjectured value is

$$
b_{0}:=\arctan (2 \sqrt{2})=\arccos (1 / 3) \approx 70^{\circ} 32^{\prime} .
$$

This is the spherical radius of the disc $|w| \leq 1 / \sqrt{2}$. The conjectured extremal function is the Weierstraß $\wp$-function satisfying

$$
\left(\wp^{\prime}\right)^{2}=4\left(\wp-e_{0}\right)\left(\wp-e_{1}\right)\left(\wp-e_{2}\right), \quad \text { where } \quad e_{j}=(1 / \sqrt{2}) \exp (2 \pi i j / 3) .
$$

Via stereographic projection the points $e_{0}, e_{1}, e_{2}$ and $\infty$ correspond to the vertices of a regular tetrahedron. This example is due to Minda [16].

We say that a meromorphic function $f: \mathbf{C} \rightarrow \overline{\mathbf{C}}$ belongs to the class $\mathcal{Y}$ (after K. Yosida, who studied this class in [25]) if the family of translates $\{f(\cdot+\lambda): \lambda \in \mathbf{C}\}$ is normal and has no constant limit functions. The class $\mathcal{Y}$ evidently contains all elliptic functions. Normal functions, i.e., meromorphic functions in $\mathbf{C}$ with bounded spherical derivative, are sometimes called Yosida functions. To avoid confusion in the present context note that every function in $\mathcal{Y}$ is a normal function and hence a Yosida function, but not necessarily vice versa.

Theorem $1.5 \mathfrak{B}_{\mathcal{Y}}=b_{0}$. 
Corollary 1.6 The spherical Bloch constant for the class of (non-constant) elliptic functions is equal to $b_{0}$.

Now we consider holomorphic maps (meromorphic functions) $f: X \rightarrow \overline{\mathbf{C}}$, where $X$ is a compact Riemann surface of genus $g$. Let us define the function

$$
b(\delta)=\left\{\begin{array}{cl}
\arccos \left(\frac{1}{\sqrt{3}} \cot \left(\frac{\pi(\delta+1)}{6}\right)\right), & \delta \in[0,2], \\
\pi / 2, & \delta \in(2, \infty] .
\end{array}\right.
$$

For $\delta \in[0,2]$ this is the circumscribed radius of an equilateral spherical triangle with spherical area $\pi \delta$. The function $b$ is non-decreasing, and $b_{0}=b(1)$.

Denote by $\mathfrak{B}_{g, d}$ the spherical Bloch constant for meromorphic functions of degree $d$ defined on a compact Riemann surface of genus $g$.

Theorem $1.7 \mathfrak{B}_{g, d} \geq b(d /(d+2 g-2)), \quad g \in \mathbf{N} \cup\{0\}, d \in \mathbf{N}, d \geq 2$.

Here we excluded the trivial case $d=1$, because if $g \geq 1$ there are no meromorphic functions with $d=1$. If $g=0$ the functions $f$ with $d=1$ are Möbius transformation and so $\mathfrak{B}_{f}=\pi$. We defined the function $b(\delta)$ for $\delta>2$ in order to cover the cases $(g, d)=(0,2),(0,3)$.

If in the theorem the surface is the Riemann sphere, then $g=0$ and we obtain

Corollary 1.8 For every rational function $f$ of degree $d \in \mathbf{N}, d \geq 2$, we have $\mathfrak{B}_{f} \geq b(d /(d-2))>b_{0}$.

Minda in [16] obtained the lower estimate $\mathfrak{B}_{\mathcal{R}} \geq 54^{\circ} 44^{\prime}$ for the class $\mathcal{R}$ of all non-constant rational functions.

Example 1.9 There exists a rational function $f$ of degree $d=7$, such that $\mathfrak{B}_{f}=b(7 / 5) \approx 79^{\circ} 11^{\prime}$.

In particular, the estimate in Corollary 1.8 is sharp for $d=7$. This example was found in collaboration with William Cherry. The best previously known upper estimate was $\mathfrak{B}_{\mathcal{R}} \leq 90^{\circ}$. We do not know whether $\mathfrak{B}_{\mathcal{R}}=b_{0}$.

Every elliptic function can be considered as a meromorphic function on a complex torus. In this case, $g=1$ and so Corollary 1.6 also follows from Theorem 1.7. When $g \geq 2$ is fixed, $b(d /(d+2 g-2))$ is an increasing function of $d$, so our best lower estimate, independent of $d$ will be $b(1 / g)$. So if we denote by $\mathfrak{B}_{g}$ the spherical Bloch constant for all meromorphic functions on Riemann surfaces of genus $g \geq 2$, then

$$
\mathfrak{B}_{g} \geq b(1 / g) .
$$


For $g=2$ and $g=5$ we have equality in (4). This is demonstrated by examples of hyperelliptic Riemann surfaces and holomorphic maps of degree 2 , ramified over the vertices of a regular octahedron for $g=2$ and a regular icosahedron for $g=5$.

Also (4) gives the right order of magnitude when $g \rightarrow \infty$. Again this can be demonstrated by holomorphic maps of degree 2 from hyperelliptic Riemann surfaces to the sphere with critical values "uniformly spread" over the sphere.

The history of our problem begins with Valiron's theorem [21] which says that for every entire function $f$ there is a Euclidean disc $D$ of arbitrarily large radius, such that a single valued branch of the inverse $f^{-1}$ exists in $D$. Valiron's theorem follows from our Theorem 1.1. Bloch [3] improved Valiron's theorem in the following way: for every holomorphic function $f$ in the unit disc satisfying $\left|f^{\prime}(0)\right|=1$ there exists a disc of Euclidean radius $R$ where a single-valued branch of $f^{-1}$ is defined. Here $R>0$ is an absolute constant. In $[15,16]$ D. Minda extended Bloch's theorem to various metrics other than the Euclidean one, and gave upper and lower estimates of the corresponding Bloch constants. The relation of our theorems 1.1 and 1.3 to functions in the unit disc will be explained in Section 2 .

Section 2 contains preliminaries for the proofs of Theorems 1.1 and 1.3. Section 3 begins with a brief explanation of the main idea of the proof of Theorem 1.1 and contains lemmas used in this proof. The proofs of these Theorems 1.1 and 1.3 are given in Sections 4 and 5, respectively. Example 1.2 is constructed in Section 6. The necessary considerations for proving Theorems 1.5 and 1.7 are given in Sections $7-10$ which are independent of the contents of Sections 2-6. The proofs are based on some propositions from spherical geometry which are contained in Section 7 and a construction of a tiling of a Riemann surface by spherical triangles, which may have independent interest, in Section 8. Finally we prove Theorem 1.5 in Section 9 and Theorem 1.7 in Section 10, which ends with Example 1.9.

The authors thank William Cherry for co-operation with Example 1.9. We also thank David Drasin, Michael Gromov, David Minda, and Allen Weitsman for stimulating discussions. We especially acknowledge the work of the referee which substantially improved the paper.

\section{Rescaling Principle}

The crucial step in our proof of Theorems 1.1 and 1.3 is the application of the following result of Zalcman [26], whose idea goes back to [14]. See also 
$[4,12,2,17,7]$ and the survey $[27]$.

Every Riemann surface $X$ carries a complete Riemannian metric of constant curvature $1,-1$ or 0 which is compatible with the conformal structure. The first case occurs when $X=\overline{\mathbf{C}}$. Then the metric is the spherical metric of curvature 1, introduced above. The other two cases occur according to whether the universal covering surface of $X$ is conformally equivalent to the unit disc (hyperbolic case) or the complex plane (parabolic case).

In the hyperbolic case, the complete metric of curvature -1 is uniquely determined, and is called the Poincaré metric. In the parabolic case, the complete metric of curvature 0 is unique up to a scaling factor. The group of isometries does not depend on the choice of this factor.

Thus for every Riemann surface $X$ the group of sense-preserving isometries $\operatorname{Iso}(X)$ is well-defined. It is a subgroup of the group $\operatorname{Aut}(X)$ of all conformal automorphisms.

Suppose that the Riemann surface $X$ is a region $G \subseteq \mathbf{C}$. A meromorphic function $f$ in $G$ is called normal ${ }^{2}$ if the family $\{f \circ \phi: \phi \in \operatorname{Iso}(G)\}$ is a normal family.

Lemma 2.1 Let $\mathcal{M}$ be a set of pairs $(G, f)$, where $G \subseteq \mathbf{C}$ is an open Euclidean disc or the complex plane, and $f$ is a meromorphic function on $G$. Assume that $\mathcal{M}$ has the following properties:

(i) If $(G, f) \in \mathcal{M}$ and $\phi$ : $G^{\prime} \rightarrow G$ is a map of the form $z \mapsto \phi(z)=a z+b$, $a \neq 0$, then $\left(G^{\prime}, f \circ \phi\right) \in \mathcal{M}$.

(ii) If $\left(G_{k}, f_{k}\right) \in \mathcal{M}$ and $G_{k} \subseteq G_{k+1}$ for $k \in \mathbf{N}, \bigcup_{k \in \mathbf{N}} G_{k}=G$, and if $\left(f_{k}\right) \rightarrow f \not \equiv$ const locally uniformly, then $(G, f) \in \mathcal{M}$.

Then the following statements about $\mathcal{M}$ are equivalent:

(1) $(\mathbf{C}, f) \in \mathcal{M}$ implies $f \equiv$ const,

(2) $(\mathbf{C}, f) \in \mathcal{M}$ and $f$ is normal imply $f \equiv$ const,

(3) For every $G$ the family $\{f:(G, f) \in \mathcal{M}\}$ is normal.

Remark 2.2 If (i) only holds for affine holomorphic maps which are bijective, then we can still conclude the equivalence of (1) and (2). [17, Theorem $1]$.

\footnotetext{
${ }^{2}$ Usually, this name is only applied to functions in the unit disc. We prefer to extend the definition to make the formulation of Lemma 2.1 more symmetric. As we remarked above, sometimes normal functions in the plane are called Yosida functions.
} 
Examples of sets $\mathcal{M}$ satisfying (i) and (ii) in Lemma 2.1 are functions which omit a given value and locally univalent functions. Notice that the intersection of two sets $\mathcal{M}_{1}$ and $\mathcal{M}_{2}$ satisfying (i) and (ii) also satisfies these conditions.

For our application of Lemma 2.1 to the proof of Theorem 1.1 we have to verify first of all that the set $\mathcal{M}$ defined by the property $\left(\mathbf{P}_{\epsilon}\right)$ in the Introduction satisfies conditions (i) and (ii) of Lemma 2.1. It is evident that (i) holds.

Property (ii) follows from the Argument Principle. Indeed, assume that all $f_{k}$ satisfy $\left(\mathbf{P}_{\epsilon}\right)$ with the same $\epsilon \in(0, \pi / 2)$, and $\left(f_{k}\right) \rightarrow f \not \equiv$ const locally uniformly, but $f$ does not satisfy $\left(\mathbf{P}_{\epsilon}\right)$. This means that there exists a disc $B$ of spherical radius $r>\pi / 2-\epsilon$ and $\operatorname{dist}(B, \infty)<\epsilon$, such that a branch $\Phi$ of $f^{-1}$ exists in $B$. Take a smaller concentric disc $B_{1}$ which still violates the conditions of $\left(\mathbf{P}_{\epsilon}\right)$, and choose a circle $C \subseteq B$ separating $B_{1}$ from $\partial B$. Applying the Argument Principle to the Jordan curve $\Phi(C)$ and to any point $a \in B_{1}$ we conclude that the functions $f_{k}$ are univalent in $\Phi\left(B_{1}\right)$ for large values of $k$. The images $f_{k}\left(\Phi\left(B_{1}\right)\right)$ tend to $B_{1}$ by the Caratheodory Kernel Theorem, so we get a contradiction which proves (ii).

For the proof of Theorem 1.3 we consider the following property:

$\left(\mathbf{Q}_{\epsilon}\right)$ No region where a single-valued branch of $f^{-1}$ exists contains a set of the form $\overline{\mathbf{C}} \backslash l_{\epsilon}$. Here $l_{\epsilon}$ is the open spherical $\epsilon$-neighborhood of an arc $l$ of a great circle, whose spherical length is at most $\pi$.

The verification that $\left(\mathbf{Q}_{\epsilon}\right)$ defines a family satisfying (i) and (ii) of Lemma 2.1 is similar to that of property $\left(\mathbf{P}_{\epsilon}\right)$.

Thus Theorem 1.3 is an immediate corollary from the following proposition, which will be proved in Section 5:

Proposition 2.3 The only normal meromorphic locally univalent functions in $\mathbf{C}$ are fractional-linear functions and exponentials, that is the functions of the form $L \circ \exp (a z)$, where $L$ is an automorphism of $\overline{\mathbf{C}}$ and $a \in \mathbf{C} \backslash\{0\}$.

We have seen that the equivalence $(1) \Leftrightarrow(3)$ gives the following normality criteria, equivalent to Theorems 1.1 and 1.3 , respectively.

Theorem 2.4 A family of holomorphic functions in a region $G$, satisfying $\left(\mathbf{P}_{\epsilon}\right)$ with fixed $\epsilon \in(0, \pi / 2)$ is normal.

Theorem 2.5 A family of locally univalent meromorphic functions in a region $G$, satisfying $\left(\mathbf{Q}_{\epsilon}\right)$ with fixed $\epsilon \in(0, \pi / 2)$ is normal. 
Corollary 2.6 [20] A locally univalent meromorphic function $f$ in the unit disc with $\mathfrak{B}_{f}<\pi / 2$ is normal.

The last result was first proved by Pommerenke.

Thus Lemma 2.1 shows that in a certain well-defined class of problems it does not matter whether we work with meromorphic functions in the plane, normal functions in the plane or normal families.

For our purposes the most important part of Lemma 2.1 is the equivalence $(1) \Leftrightarrow(2)$. It permits the reduction of theorems 1.1 and 1.3 to their special cases for normal functions in the plane. Normal functions in $\mathbf{C}$ have finite order.

We recall the definition of asymptotic curves and asymptotic values. Let $X$ be an open Riemann surface, that is a surface which is not compact, and let $f: X \rightarrow \overline{\mathbf{C}}$ be a holomorphic map. Define $\bar{X}:=X \cup\{\infty\}$ to be the onepoint compactification of $X$. Here $\infty$ is the point at infinity. This notation is ambiguous, if $X$ is a subregion of $\overline{\mathbf{C}}$ containing $\infty \in \overline{\mathbf{C}}$, but in the context of asymptotic curves and values $\infty$ will always be the point at infinity of the one-point compactification. An asymptotic curve $\gamma:[0,1) \rightarrow X$ is a curve with the properties $\gamma(t) \rightarrow \infty$ and $f \circ \gamma(t) \rightarrow a \in \overline{\mathbf{C}}$ for $t \rightarrow 1$. A point $a \in \overline{\mathbf{C}}$ for which such a curve exists is called an asymptotic value. Notice that by definition meromorphic functions on compact Riemann surfaces have no asymptotic curves and asymptotic values.

Proposition 2.7 Normal meromorphic functions in $\mathbf{C}$ have order at most 2 , normal type, that is $T(r, f)=O\left(r^{2}\right), r \rightarrow \infty$, where $T$ is the Nevanlinna chatacteristic. Normal entire functions have at most exponential type. In particular, normal entire functions have at most two finite asymptotic values.

The first statement is due to K. Yosida [25]. Normal meromorphic functions in $\mathbf{C}$ are exactly those whose spherical derivative is bounded in $\mathbf{C}$. So the first statement of Proposition 2.7 follows from Ahlfors-Shimizu form for the Nevanlinna characteristic (see Section 9). The truth of the second statement (which is much deeper) was discovered by Clunie and Hayman in [8]. A simpler proof, which also gives precise estimates was derived by Minda [17] from a result of Pommerenke [20]. The last statement, about asymptotic values, follows from the

\section{Denjoy-Carleman-Ahlfors Theorem}

(i) An entire function of order $\rho$ has at most $2 \rho$ finite asymptotic values.

(ii) For an entire function of order $\rho$ the sets $\{z:|f(z)|>c\}, c \geq 0$, have at most $\max \{2 \rho, 1\}$ components. 
See, for example, [10].

Proposition 2.7 shows that it is enough to prove Theorem 1.1 for functions of exponential type. We need one more reduction.

Lemma 2.8 If there exists a non-constant entire function with the property $\left(\mathbf{P}_{\epsilon}\right)$, then there exists a non-constant entire function $f$ of exponential type satisfying $\left(\mathbf{P}_{\epsilon / 2}\right)$ with the following additional properties:

(i) the point 0 is neither a critical value nor asymptotic value,

(ii) every ray starting from 0 contains at most one critical or asymptotic value,

(iii) all critical points are simple, and critical values corresponding to different critical points are distinct,

(iv) no critical value is equal to an asymptotic value.

Proof. Let $g_{0} \neq$ const be an entire function with the property $\left(\mathbf{P}_{\epsilon}\right)$. First we find, using Lemma $2.1,2 \Rightarrow 1$ a normal entire function $g_{1} \neq$ const satisfying $\left(\mathbf{P}_{\epsilon}\right)$. By Proposition $2.7 g_{1}$ may have at most two finite asymptotic values. We add, if necessary, to $g_{1}$ a small constant (of absolute value less than $\epsilon / 4$ ) to make sure that 0 is not an asymptotic value and that there is no pair of asymptotic values belonging to a ray from 0 . Let the new function be $g_{2}$; it evidently satisfies $\left(\mathbf{P}_{3 \epsilon / 4}\right)$.

Now it remains to make a perturbation of critical points. This can be done with a well-known perturbation argument, based on Teichmüller's distortion theorem (see, for example, $[24, \mathrm{Ch} . \mathrm{VI}]$ or $[13, \mathrm{Ch} . \mathrm{V}, \S 6]$ ). Here is a more elementary proof. Let $\left(z_{k}\right)$ be a sequence of isolated points in $\mathbf{C}$. Then there exist positive numbers $\rho_{k}$ such that every function $\phi$ holomorphic and univalent in $\mathbf{C} \backslash \bigcup_{k}\left\{z:\left|z-z_{k}\right|<\rho_{k}\right\}$ satisfies there $|L(\phi(z))-z|<1$, for some $L(z)=a z+b, a \neq 0$ (see [5, Theorem 13], where this is proved with a simple normality argument).

For $\left(z_{k}\right)$ we take an enumeration of all the critical points of $g_{2}$ and find the corresponding sequence $\left(\rho_{k}\right)$. Then we modify $g_{2}$ in the discs $D_{k}:=\left\{z:\left|z-z_{k}\right|<\rho_{k}\right\}$ so that the new function $g^{*}$ is topologically holomorphic, coincides with $g_{2}$ outside the union of the discs $D_{k}$, has the desired properties for its critical values and satisfies $\left(\mathbf{P}_{\epsilon / 2}\right)$. The asymptotic values remain unchanged at this stage. This new function $g^{*}$ will be analytic with respect to some new conformal structure in the plane. This means that there is a homeomorphism $\phi: \mathbf{C} \rightarrow X$, where $X$ is either $\mathbf{C}$ or the unit disk, such that $g^{*} \circ \phi^{-1}$ is holomorphic on $X$. It is clear that $\phi$ is holomorphic 
in the complement of $\bigcup_{k} D_{k}$, thus the statement above shows that $X=\mathbf{C}$, so that $f=g^{*} \circ \phi^{-1}$ is an entire function. Since this function has the same critical and asymptotic values as $g^{*}$, the critical values of $f$ satisfy all our requirements. Moreover, $f$ satisfies $\left(\mathbf{P}_{\epsilon / 2}\right)$. Finally, $g_{2}$ was of exponential type, thus $f$ will also be of exponential type because, $\phi(z)=a z+O(1)$ and $a \neq 0$.

\section{Outline and beginning of proof of Theorem 1.1. Star decomposition}

In sections 3 and 4 we consider an entire function $f \neq$ const of exponential type satisfying $\left(\mathbf{P}_{\epsilon}\right)$. We assume in addition that the critical points of $f$ satisfy all the conditions of Lemma 2.8. Our purpose is to show that such a function does not exist.

The proof of Theorem 1.1 is somewhat technical, but the idea behind it is very simple. We start with an outline of the proof, assuming for simplicity that there are no finite asymptotic values. We consider all germs of the inverse $f^{-1}$ at 0 . To each such germ corresponds a maximal domain starlike with respect to 0 to which the germ can be analytically continued. We call these domains stars. The $f$-preimages of stars make a tiling of the plane. Every star $H$ is obtained from the plane by deleting a certain set of rays which is at most countable. Each ray starts at a critical value of $f$. As we assume that there are no finite asymptotic values, the rays of a single star do not accumulate. The stars are "attached" to each other along these rays. The preimages of two attached stars have a common simple boundary curve, tending to infinity in both directions. It is easy to see that the preimages of the stars are attached to each other in a tree-like pattern. If we consider a graph whose vertices correspond to stars and edges to common boundary curves of pairs of stars, this graph will be a tree (with all branches infinite). Now, if $f$ satisfies $\left(\mathbf{P}_{\epsilon}\right)$, no star can contain large spherical discs. Elementary geometry (Lemma 3.4) shows that each star has at least three complementary rays, all three intersecting some fixed circle $|w|=r_{1}$. Thus every vertex in our tree has at least three outgoing edges. In particular, it contains the so-called "modular figure", the regular tree with vertex degree 3. The "modular pattern" is hard to reconcile with parabolicity, but can sometimes occur (see Example 1.2). To get a contradicition it is important that all complementary rays of stars which correspond to our modular figure intersect the same circle $|w|=r_{1}$. This permits us to construct a lot of curves tending to infinity on which $f$ is bounded. On the other hand, these 
curves can be separated by other curves, on which $f$ tends to infinity. This contradicts the Denjoy-Carleman-Ahlfors Theorem (ii). The only technical difficulty is due to the possible presence of finite asymptotic values, which may spoil the picture.

Now we proceed to the formal proof. We choose

$$
r_{1}>\tan (\pi / 2-\epsilon / 3),
$$

such that all finite asymptotic values of $f$ are contained in the disc $D\left(0, r_{1}\right)$ and there are no critical values on the circle $C:=\left\{w:|w|=r_{1}\right\}$. The number $r_{1}$ and the circle $C$ will be fixed until the end of the proof.

Consider a germ $\phi$ of $f^{-1}$ at the point 0 . The star $H=H_{\phi}$ is defined as the maximal domain starlike (in Euclidean sense) with respect to 0 into which analytic continuation of $\phi$ is possible. We call the result of this continuation $\Phi$. Then $\Phi$ is a holomorphic function in $H$, namely the branch of $f^{-1}$ in $H$, and it is univalent.

We can consider each of these functions as the restriction to the stars of one single holomorphic function, also called $\Phi$ by abuse of notation, defined on the disjoint union of all stars $H$. This function $\Phi$ is still univalent. The simply connected unbounded regions $G:=\Phi(H) \subseteq \mathbf{C}$ can be labeled by solutions of the equation $f(z)=0$; regions $G$ corresponding to different solutions are disjoint. Sometimes the regions $H$ or $G$ are called Gross stars corresponding to $f$. We first study the properties of a single star $H=H_{\phi}$ for an arbitrary fixed germ $\phi$.

For every ray $l=t e^{i \theta_{0}}, 0 \leq t<\infty$, we have the following possibilities:

(i) Analytic continuation of $\phi$ is possible along the whole ray $l$.

In this case $l \subseteq H$.

(ii) Analytic continuation of $\phi$ along $l$ is possible for $0 \leq t<t_{0}$, but

$$
\lim _{t \rightarrow t_{0}-} \phi\left(t e^{i \theta_{0}}\right)=\infty
$$

In this case the ray $L\left(\theta_{0}\right):=\left\{t_{0} e^{i \theta_{0}}: t \geq t_{0}\right\} \subseteq \mathbf{C} \backslash H$ will be called an asymptotic ray. Each star can have at most two asymptotic rays in its complement, because we assume that the function $f$ is of exponential type, hence it has at most two finite asymptotic values by the Denjoy-CarlemanAhlfors Theorem.

(iii) Analytic continuation of $\phi$ along $l$ is possible for $0 \leq t<t_{0}$, but not further, and

$$
\lim _{t \rightarrow t_{0}-} \phi\left(t e^{i \theta_{0}}\right)=z_{0} \in \mathbf{C} .
$$


In this case $z_{0}$ is a critical point and the ray $L\left(\theta_{0}\right)$ in the complement of $H$ will be called a critical ray.

That (i), (ii) and (iii) exhaust all possibilities follows from Iversen's theorem (see, for example, [18]), which we state here for the reader's convenience.

Iversen's Theorem Let $X$ be a Riemann surface, $f: X \rightarrow \overline{\mathbf{C}}$ a holomorphic map, and $p_{0} \in X$ a point which is not critical. Let $w_{0}=f\left(p_{0}\right)$, and assume that $\phi$ is a germ of $f^{-1}$ with $\phi\left(w_{0}\right)=p_{0}$, and $\gamma:[0,1] \rightarrow \overline{\mathbf{C}}$ is a curve with $\gamma(0)=w_{0}$.

If analytic continuation of $\phi$ along $\gamma \mid[0, t]$ is possible for $t \in[0,1)$, but impossible for $t=1$, then either $\phi(\gamma(t))$ has a limit $p_{1} \in X$ as $t \rightarrow 1$, and $p_{1}$ is a critical point, or $\phi(\gamma(t))$ leaves every compact subset of $X$ as $t \rightarrow 1$. In the second case $\phi(\gamma)$ is an asymptotic curve with asymptotic value $\gamma(1)$.

We need more details about the structure of the boundary $\partial H$. First we consider a critical ray starting at $w_{0}=f\left(z_{0}\right)=t_{0} e^{i \theta_{0}}$.

Lemma 3.1 Critical rays are isolated. That is, for every critical ray $L$ there exists a neighborhood $U$ such that $U \backslash L \subseteq H$. Thus to every point of $L$ except $w_{0}$, correspond exactly two accessible boundary points of $H$. The function $\Phi$ has direct analytic continuation to every accessible boundary point of $L \backslash\left\{w_{0}\right\}$.

Proof. There is a neighborhood $V$ of $z_{0}$ such that $D:=f(V)$ is a Euclidean disc centered at $w_{0}$ and

$$
f: V \backslash\left\{z_{0}\right\} \rightarrow D \backslash\left\{w_{0}\right\}
$$

is a covering. Let $D_{1}$ be the disc concentric with $D$ and of half its radius. We can find $\delta>0$ and $t_{1} \in\left(0, t_{0}\right)$ such that the $\operatorname{arc}\left\{t_{1} e^{i \theta}:\left|\theta-\theta_{0}\right| \leq \delta\right\}$ is contained in $D_{1}$ and such that the germ $\phi$ has analytic continuation along the segments $l_{\theta}:=\left\{t e^{i \theta}: 0 \leq t \leq t_{1}\right\}$ for $\theta \in\left[\theta_{0}-\delta, \theta_{0}+\delta\right]$. Since in (6) we have a covering, the continuation is also possible along the extended segments $l_{\theta}^{\prime}:=\left\{t e^{i \theta}: 0 \leq t \leq t_{2}\right\}, 0<\left|\theta-\theta_{0}\right| \leq \delta$, where $t_{2}>t_{0}$. The extended rays $l_{\theta}^{\prime}$ fill the set $\left\{t e^{i \theta}: 0 \leq t \leq t_{2}, 0<\left|\theta-\theta_{0}\right| \leq \delta\right\} \cup\left(0, w_{0}\right)$ which thus belongs to $H$. Now we can perform the analytic continuation of $\phi$ along the following path: first we take the segment $\left[0, t_{2} e^{i\left(\theta_{0}-\delta\right)}\right]$, then follow the $\operatorname{arc}\left\{t_{2} e^{i \theta}: \theta_{0}-\delta \leq \theta \leq \theta_{0}\right\}$ to the ray $L$ and then follow $L$ to infinity. This is possible because $L$ contains no critical or asymptotic values except at its starting point, by Lemma 2.8 (ii). Similarly, we perform analytic continuation of $\phi$ to the other "side" of $L$ by following first $\left[0, t_{2} e^{i\left(\theta_{0}+\delta\right)}\right]$, 
then the $\operatorname{arc}\left\{t_{2} e^{i \theta}: \theta_{0}+\delta \geq \theta \geq \theta_{0}\right\}$ to $L$ and then $L$ to infinity. All statements of the lemma immediately follow from this.

As was said above, the images $\Phi(H)$ of different stars are disjoint. We will investigate how these images fill the plane. For a finite asymptotic value $a$ consider the set $\{z \in \mathbf{C}: \arg (f(z)-a)=\arg (a)\}$. From Lemma 2.8 (ii) follows that there are no critical points on this set, so it consists of an at most countable union of simple analytic curves which do not accumulate (that is, each curve has a neighborhood such that all these neighborhoods are disjoint). Each curve of our set is mapped by $f$ homeomorphically onto the ray $\{a+t \exp (i \arg (a)): t>0\}$. There are curves of two kinds: some of them may have an endpoint at an $a$-point of $f$, others tend to infinity in both directions. The curves of the second kind will be called the exceptional curves and their union (over all finite asymptotic values) the exceptional set $E$. Notice that for every star $H$ we have $\Phi(H) \cap E=\emptyset$. One half of every exceptional curve is an asymptotic curve with finite asymptotic value.

Lemma 3.2 Every point in $\mathbf{C} \backslash E$ has a neighborhood $V$ which intersects one or two images $G=\Phi(H)$ of stars $H$.

Proof. Let $z_{0}$ be a non-exceptional point. Assume first that $f^{\prime}\left(z_{0}\right) \neq 0$. Put $w_{0}=f\left(z_{0}\right)$ and consider the germ $\phi_{0}$ of $f^{-1}$ with $\phi_{0}\left(w_{0}\right)=z_{0}$. We try to continue $\phi_{0}$ analytically along the segment $l=\left\{w_{0}(1-t): 0 \leq t \leq 1\right\}$. If such continuation exists, consider its result, a germ $\phi$ at 0 . Evidently in this case $w_{0} \in H_{\phi}$ and $\Phi\left(H_{\phi}\right)$ is a neighborhood of $z_{0}$. Thus $z_{0}$ has a neighborhood which intersects only one star image, namely $\Phi\left(H_{\phi}\right)$.

If the continuation along $l$ is impossible, this may happen for two reasons. For some $t_{1} \in(0,1)$ either

(i) $\phi_{0}\left(w_{0}(1-t)\right) \rightarrow \infty$ as $t \rightarrow t_{1}-0$ or

(ii) $\phi_{0}\left(w_{0}(1-t)\right) \rightarrow z_{1} \in \mathbf{C}$ as $t \rightarrow t_{1}-0$.

In case (i), the point $z_{0}$ is exceptional, contrary to our assumption.

In case (ii) we conclude that $z_{1}$ is a critical point. Note that $w_{1}:=f\left(z_{1}\right)$ is the only critical value on the segment $l$, and there are no asymptotic values on this segment. This follows from Lemma 2.8 (ii). We modify the segment $l$ by replacing a small interval whose midpoint is $w_{1}$ by a semi-circle which has this interval as diameter. Depending on which of the two semicircles we take, we obtain two modified paths, $l^{+}$and $l^{-}$, such that analytic continuation along these paths is possible. The results of these continuations are two distinct germs $\phi^{ \pm}$at 0 . Now as in the proof of Lemma 3.1 we show 
that $z_{0}$ has a neighborhood $V$ which intersects $\Phi\left(H_{\phi^{+}}\right)$and $\Phi\left(H_{\phi^{-}}\right)$and the union of these intersections is dense in $V$. So $V$ cannot intersect the image of any other star.

If $f^{\prime}\left(z_{0}\right)=0$, we consider a neighborhood $V$ of $z_{0}$ such that (6) is a covering and $D$ is an open Euclidean disc. Then pick a point $w_{1} \in D$ which lies on the segment connecting $w_{0}$ to 0 . There are exactly two germs at $w_{1}$ whose values belong to $V$. Analytic continuation of these germs along the segment $\left[w_{1}, 0\right]$ is possible because this segment contains no critical values and no asymptotic values. This continuation gives us two germs $\phi^{+}$and $\phi^{-}$at 0 . It is clear now that a neighborhood of $z_{0}$ intersects $\Phi\left(H_{\phi^{+}}\right)$and $\Phi\left(H_{\phi^{-}}\right)$and no $\Phi$-images of other stars.

Let $H_{0}$ be a star, $L$ a critical ray complementary to $H_{0}$, and $w_{0}$ the initial point of $L$. A boundary value $z$ of $\Phi$ at an accessible point of $L \backslash\left\{w_{0}\right\}$ is non-exceptional, so there is a neighborhood of $z$ intersecting the images of exactly two stars, $H_{0}$ and $H_{1}$. Our assumption that all critical points are simple, and all critical values lie on distinct rays from 0 , implies that $H_{1}$ does not depend on the choice of the accessible boundary point on $L$. We say that $H_{0}$ and $H_{1}$ are attached to each other along the critical ray $L$. The domains $G_{0}=\Phi\left(H_{0}\right)$ and $G_{1}=\Phi_{1}\left(H_{1}\right)$ have a common boundary curve $\Gamma$ - the image of $L$ under the extension of $\Phi$ to the accessible boundary points of $L$. This is a simple analytic curve tending to infinity in both directions. Thus it divides the plane into two regions, say $\Omega_{0}$ and $\Omega_{1}$ such that $G_{j} \subseteq \Omega_{j}$. We have

Lemma 3.3 Let $L_{1}$ and $L_{2}$ be two different critical rays complementary to a star $H_{0}$. Then the stars $H_{1}$ and $H_{2}$ attached to $H_{0}$ along $L_{1}$ and $L_{2}$ are different and different from $H_{0}$. Furthermore, $G_{0}=\Phi\left(H_{0}\right)$ separates $G_{1}=\Phi_{1}\left(H_{1}\right)$ from $G_{2}=\Phi_{2}\left(H_{2}\right)$.

"Separates" means that $G_{1}$ and $G_{2}$ are contained in different components of the complement $\mathbf{C} \backslash G_{0}$.

Proof. We only have to prove that $G_{1}$ and $G_{2}$ are separated by $G_{0}$. Consider two rays $l_{1}$ and $l_{2}$ starting at 0 and completely contained in $H_{0}$, such that the simple curve $l:=l_{1} \cup l_{2}$ separates the rays $L_{1}$ and $L_{2}$ in $\mathbf{C}$. Let the starting points of $L_{j}$ be $w_{j}, j \in\{1,2\}$. Then $\gamma^{\prime \prime}:=\left[w_{1}, 0\right] \cup\left[0, w_{2}\right]$, intersects $l$ transversally at the single point 0 . The image curve $\Gamma=\Phi(l)$ tends to infinity in both directions, because $f$ is entire, so by the Jordan theorem (applied in $\overline{\mathbf{C}}$ ), it separates the plane. If $G_{1}$ and $G_{2}$ were in the same component $\Omega$ of $\mathbf{C} \backslash \Gamma$, their boundaries would also belong to this component. In this case the points $\Phi\left(w_{j}\right), j \in\{1,2\}$, are in $\Omega$. We connect 
these points by a curve $\gamma^{\prime} \subseteq \Omega$, so that $\gamma:=\gamma^{\prime} \cup \Phi\left(\gamma^{\prime \prime}\right)$ is a closed curve. Thus two closed curves $\Gamma$ and $\gamma$ on the sphere $\overline{\mathbf{C}}$ intersect transversally at a single point, which is impossible. Thus $G_{1}$ and $G_{2}$ are separated by $\Gamma$ and so they are separated by $G_{0}$.

Now we draw conclusions from the property $\left(\mathbf{P}_{\epsilon}\right)$ which we assume our function $f$ satisfies.

Lemma 3.4 If $f$ satisfies $\left(\mathbf{P}_{\epsilon}\right)$ then each star has at least three complementary rays intersecting the circle $C=\left\{w:|w|=r_{1}\right\}$, where $r_{1}$ was chosen in (5)

Proof. Assume that for some star $H$ at most two complementary rays intersect $C$. If there are exactly two, let $\alpha \in \mathbf{C},|\alpha|=1$ be such that $\arg \alpha$ bisects the smaller angle between two rays. If there is only one ray, let $\arg \alpha$ be the direction of this ray. If there is no complementary ray intersecting $C$ we choose arbitrary $\arg \alpha$. In any case the half-plane $Q:=\{\alpha w: \operatorname{Re} w<0\}$ does not intersect these (one or two) rays, and thus the half-disc $S=Q \cap\{w:$ $\left.|w|<r_{1}\right\}$ is contained in $H$. Using (5) we obtain the following lower bound for the spherical diameter of the spherical disc inscribed into $S$

$$
\int_{0}^{r_{1}} \frac{2 d t}{1+t^{2}}=2 \arctan \left(r_{1}\right)>\pi-\epsilon
$$

The spherical distance from this inscribed disc to infinity is bounded by

$$
\int_{r_{1}}^{\infty} \frac{2 d t}{1+t^{2}}<\pi-\pi+2 \epsilon / 3<\epsilon
$$

Thus $f$ does not satisfy $\left(\mathbf{P}_{\epsilon}\right)$.

As a corollary we obtain that every star must have at least three isolated rays in its complement. Indeed, the number of rays is at least three by Lemma 3.4. If one of them in not isolated, then there are in fact infinitely many rays. But at most two of them can be non-isolated, because nonisolated rays have to be asymptotic and $f$ has at most two finite asymptotic values.

\section{Conclusion of the proof of Theorem 1.1}

Recall our standing assumption: $f \neq$ const is an entire function of exponential type, satisfying all conditions of Lemma 2.8, and the assumption $\left(\mathbf{P}_{\epsilon}\right)$ 
from the Introduction. We are trying to prove that such function cannot exist. The positive number $r_{1}$ and the circle $C$ were chosen in the beginning of the proof. Notations and results from the previous Section 3 are used.

We start with an arbitrary star which will be called $H_{0}$. Let $L_{0}, L_{1}$, and $L_{2}$ be isolated rays complementary to $H_{0}$. They are enumerated in anti-clockwise direction (the direction of increase of the argument). Now we choose three rays $l_{0}, l_{1}$ and $l_{2}$ starting from 0 , which are completely contained in $H_{0}$. We choose them in such a way that the order is the following:

$$
l_{0}, L_{0}, l_{1}, L_{1}, l_{2}, L_{2} \text {. }
$$

Put $T=\Phi\left(l_{0} \cup l_{1} \cup l_{2}\right)$. This is a "triode" in the plane consisting of three simple analytic curves, each connecting some point $z_{0}$ with infinity, and pairwise disjoint except for this point $z_{0}$. We have

$$
f(z) \rightarrow \infty \quad \text { as } \quad z \rightarrow \infty, \quad z \in T .
$$

The triode $T$ separates the plane into three regions, say $\Omega_{0}, \Omega_{1}$, and $\Omega_{2}$, enumerated anticlockwise such that $\partial \Omega_{0}=\Phi\left(l_{0} \cup l_{1}\right)$.

We are going to prove that each of these regions $\Omega_{j}$ contains a curve $\Gamma$ tending to infinity such that $f$ is bounded on $\Gamma$. This will lead to a contradiction by the Denjoy-Carleman-Ahlfors Theorem [10].

Consider any of the three regions $\Omega_{j}$. It contains the image $L_{j}$ under the boundary values of $\Phi_{0}:=\Phi \mid H_{0}$. If $\Omega_{j}$ contains an asymptotic curve with finite asymptotic value, we are done. So it is enough to consider the case when $\Omega_{j}$ does not contain an asymptotic curve with finite asymptotic value and to prove that it contains a curve tending to infinity on which $f$ is bounded.

Without loss of generality we assume that $\Omega_{0}$ does not contain asymptotic curves with finite asymptotic values. Then $L_{0}$ is a critical ray, and $\Omega_{0}$ contains the image of $L_{0}$ under the boundary extension of $\Phi_{0}$. Let $H_{1}$ be the star attached to $H_{0}$ along $L_{0}$. The image $G_{1}:=\Phi\left(H_{1}\right)$ is contained in $\Omega_{0}$, because $G_{1} \cap \partial \Omega_{0}=\emptyset$, and $G_{1}$ has boundary points in $\Omega_{0}$, so $H_{1}$ has no asymptotic complementary rays. So all complementary rays of $H_{1}$ are critical and thus isolated. By Lemma 3.4 at least three of them, say $L_{1,0}, L_{1,1}$ and $L_{1,2}$ intersect the circle $C$ (we list them in anti-clockwise order). Assume without loss of generality that $H_{1}$ is attached to $H_{0}$ along $L_{1,2}$, which is thus identified with $L_{0}$. The total number of complementary rays to $H_{1}$ which intersect $C$ is finite (because all complementary rays to $H_{1}$ are isolated), so we can find a point $w_{0} \in C=\left\{w:\left|w_{0}\right|=r_{1}\right\}$, such that the arc of the circle $C$, starting from $w_{0}$ and going to $L_{1,0}$ in anticlockwise 
direction, does not meet any complementary rays to $H_{1}$ until it hits $L_{1,0}$. Consider the curve $\gamma(t)=w_{0} e^{i t}: 0 \leq t<\infty$. Analytic continuation of any branch of $f^{-1}$ along this curve is possible because the circle $C$ contains no critical and no asymptotic values. We continue along this curve the branch $\Phi_{1}:=\Phi \mid H_{1}$. Put $\Gamma(t)=\Phi \circ \gamma(t)$. Then $|f \circ \Gamma(t)|=r_{1}$ for $0 \leq t<\infty$, so $f$ is bounded on $\Gamma$. We claim that $\Gamma(t) \rightarrow \infty$ as $t \rightarrow \infty$ and $\Gamma(t) \in \Omega_{0}$.

We have $\Gamma(0) \in G_{1}=\Phi\left(H_{1}\right)$. At some moment $t_{1}>0\left(t_{1}\right.$ is the smallest value of $t$ such that $\left.\gamma(t) \in L_{1,0}\right)$ the curve $\Gamma$ leaves $G_{1}$ and passes to $G_{2}:=\Phi\left(H_{2}\right)$ for some star $H_{2}$. This star $H_{2}$ is attached to $H_{1}$ along $L_{1,0}$, so in particular $H_{2} \neq H_{0}$, thus $G_{2} \neq G_{0}$. We have $G_{2} \subseteq \Omega_{0}$, because $G_{1} \subseteq \Omega_{0}$ and $G_{1}$ is connected with $G_{2}$ by a curve which does not intersect $G_{0} \supseteq \partial \Omega_{0}$. As $G_{2} \subseteq \Omega_{0}$ there are no asymptotic rays in $H_{2}$, thus by Lemma 3.4, $H_{2}$ has at least three critical rays intersecting $C$. Hence there is a moment $t_{2}>t_{1}, t_{2}-t_{1}<2 \pi$ when $\Gamma$ leaves $G_{2}$ and passes to some $G_{3}=\Phi\left(H_{3}\right)$. Continuing in such a way we obtain a sequence of stars $H_{k}$ with their images $G_{k}=\Phi\left(H_{k}\right)$ and an increasing sequence of values of parameters $t_{k}$ such that $t_{k+1}-t_{k}<2 \pi$ and $\Gamma\left(\left(t_{k}, t_{k+1}\right)\right) \subseteq G_{k+1}$ for $k \in \mathbf{N}$.

We claim that all the regions $G_{k}, k \in \mathbf{N}_{0}$, are distinct. To prove this assume that for some $m>n$ we have $G_{m}=G_{n}$, and assume that the difference $m-n$ is the smallest for which such equality occurs. By Lemma $3.3 m-n \geq 3$. Again by Lemma 3.3, $G_{m}=G_{n}$ separates $G_{n+1}$ from $G_{m-1}$. On the other hand, there is a curve passing from $G_{n+1}$ to $G_{n+2}$ to ... to $G_{m-1}$ without ever hitting $G_{m}$. This contradiction proves our claim that all regions $G_{k}$ are distinct.

In particular, they are all distinct from $G_{0}$, so they never intersect the boundary of $\Omega_{0}$. Thus $\Gamma(t)$ always remains in $\Omega_{0}$. Now we are ready to show that $\Gamma$ tends to infinity. Assume that there is a finite limit point $z_{0}$. Then we can find a sequence $\left(s_{j}\right)$ such that

$$
s_{j+1}-s_{j}>2 \pi
$$

$x_{j}:=\Gamma\left(s_{j}\right) \in \bigcup_{k=0}^{\infty} G_{k}$ and $\lim _{j \rightarrow \infty} \Gamma\left(s_{j}\right)=z_{0}$. In view of (8) two points $x_{j}$ and $x_{m}$ with $j \neq m$ cannot belong to the same $G_{k}$. It follows that every neighborhood of $z_{0}$ intersects infinitely many different regions $G_{k}$. Then by Lemma $3.2, z_{0} \in E$, and $z_{0}$ belongs to an exceptional curve. This exceptional curve has to lie completely in $\Omega_{0}$ because by a remark preceding Lemma 3.2 exceptional curves cannot intersect any domain $G_{k}$, in particular an exceptional curve cannot intersect $G_{0}$ (which contains the boundary of $\left.\Omega_{0}\right)$. But an exceptional curve cannot lie in $\Omega_{0}$ because every exceptional curve contains an asymptotic curve with finite asymptotic value while $\Omega_{0}$ does not, by assumption. This completes the proof of Theorem 1.1. 


\section{Proofs of Proposition 2.3 and Theorem 1.3}

It is enough to prove Proposition 2.3.

Proof. Suppose $f$ is a normal locally univalent meromorphic function. Let

$$
S[f]:=\frac{f^{\prime \prime \prime}}{f^{\prime}}-\frac{3}{2}\left(\frac{f^{\prime \prime}}{f^{\prime}}\right)^{2}=P
$$

be the Schwarzian derivative of $f$. As $f$ is locally univalent, $P$ is an entire function (see, for example, $[11,10.1]$ ). An application of Proposition 2.7, combined with the Lemma on Logarithmic Derivative [18] shows that $P$ has polynomial growth. Thus $P$ is a polynomial. If $P \equiv$ const, the only solutions of the differential equation (9) are exponential and fractional-linear functions. So it is enough to prove that $P \equiv$ const.

Assume that $P \not \equiv$ const, then

$$
P(z) \rightarrow \infty, \quad \text { as } \quad z \rightarrow \infty .
$$

All solutions of differential equations (9) have order $(\operatorname{deg} P) / 2+1$, normal type (see, for example, [24, Ch. 5 , p. 69] or $[11,5.4,10.1]$ ), so our assumption that $\operatorname{deg} P \geq 1$ implies that $f$ is at least of order $3 / 2$, normal type. The Ahlfors-Shimizu form of the Nevanlinna characteristic then shows that

$$
\limsup _{z \rightarrow \infty}|z|^{1 / 4} f^{\#}(z)>0 \quad \text { (may be infinite). }
$$

Set $M(r):=\sup \left\{f^{\#}(z):|z| \geq r\right\}$. Then $M(r)<\infty$ because $f$ is normal, so $f^{\#}$ is bounded. We claim that there is an infinite set $A$ of integers such that

$$
M\left(2^{n-1}\right) \leq 2 M\left(2^{n}\right) \quad \text { and } \quad M\left(2^{n}\right) \geq 2^{-n} \quad \text { for } \quad n \in A .
$$

To prove our claim we assume the contrary, that is for every $n \geq n_{0}$ we have either $M\left(2^{n}\right)<(1 / 2) M\left(2^{n-1}\right)$ or $M\left(2^{n}\right)<2^{-n}$. This implies that for all $n>n_{0}$ we have $M\left(2^{n}\right)<$ const $2^{-n}$, which contradicts (11). Thus the existence of an infinite set $A \in \mathbf{N}$, for which the simultaneous inequalities (12) hold, is granted.

For each $n \in A$ we choose a point $z_{n}$ with the following properties:

$$
\left|z_{n}\right| \geq 2^{n} \quad \text { and } \quad f^{\#}\left(z_{n}\right) \geq \frac{1}{2} M\left(2^{n}\right), \quad n \in A .
$$

Our function $f$ has bounded spherical derivative, so

$$
\rho_{n}:=\frac{1}{f^{\#}\left(z_{n}\right)} \geq \delta>0, \quad n \in A .
$$


From our definitions, (13), and (12) follows that

$$
\rho_{n} \leq 2^{n+1} \quad \text { and } \quad f^{\#}(z) \leq 4 / \rho_{n} \quad \text { for } \quad\left|z-z_{n}\right|<2^{n-1}, \quad n \in A .
$$

Now we put $g_{n}(z)=f\left(\rho_{n} z+z_{n}\right),|z|<1 / 4$, so that $g^{\#}(z)=\rho_{n} f^{\#}\left(\rho_{n} z+z_{n}\right)$. Using (14) and the second inequality in (15) we conclude that

$$
g_{n}^{\#}(0)=1 \quad \text { and } \quad g_{n}^{\#}(z) \leq 4 \text { for }|z|<1 / 4, n \in A .
$$

Thus $\left\{g_{n}: n \in A\right\}$ is a normal family in $D(0,1 / 4)$, and has no constant limit functions. On the other hand, (10) and (14) imply $S\left[g_{n}\right](z)=\rho_{n}^{2} S[f]\left(\rho_{n} z+\right.$ $\left.z_{n}\right)=\rho_{n}^{2} P\left(\rho_{n} z+z_{n}\right) \rightarrow \infty$ uniformly in $D(0,1 / 4)$ as $n \rightarrow \infty$. This is a contradiction.

\section{Construction of Example 1.2}

Lemma 6.1 Every disc in $\mathbf{C}$ of spherical radius $\pi / 2$ completely contains one of the intervals $l_{j}:=(0, \exp (2 \pi i j / 3)), j \in\{0,1,2\}$.

Proof. Let $B \subseteq \mathbf{C}$ be a disc of spherical radius $\pi / 2$. If $0 \notin B$ then $B$ is a half-plane and $0 \in \partial B$, and in this case the statement of Lemma 6.1 is clear. If $0 \in B$, we note that the Euclidean radius of $B$ is always at least 1 . Every Euclidean disc $D(a, r)$ with radius $r \geq 1$, containing zero, must contain in its closure at least one of the points $a_{j}:=\exp (2 \pi i j / 3), j \in\{0,1,3\}$. To see this note that the three simultaneous inequalities $\left|a-a_{j}\right|>1$ imply $|a|>1$. The statement follows.

Now we describe a construction of an open simply connected surface $X$ and a topologically holomorphic map $g: X \rightarrow \mathbf{C}$, due to L. I. Volkovyskii [23].

Fix a decreasing sequence of numbers $0<\rho_{k}<1, k \in \mathbf{N}$, to be specified later. Let $Z$ be the set consisting of the symbol 0 and all finite sequences of 1 's and 2's. An element of this set will be denoted by $K$. If $K \neq 0$ we denote by $|K|$ the length of the sequence $K$. If $K=0$ we put $|K|=0$. Consider the product $Z \times \mathbf{C}$ and denote by $g$ the projection on the second factor. The product consists of infinitely many copies of the plane $\mathbf{C}_{K}$ enumerated by elements of $Z$. On each $\mathbf{C}_{K}$ with $K=k_{1} \ldots k_{|K|} \neq 0$ we mark three points $w_{K}^{j}, j \in\{0,1,2\}$, by the following rules

$$
w_{K}^{j}=\rho_{|K|} \exp \left\{\frac{2 \pi i}{3}\left(j+\sum_{n=1}^{|K|} k_{n}\right)\right\} \quad \text { for } \quad j \in\{1,2\}
$$


and

$$
w_{K}^{0}=\rho_{|K|-1} \exp \left\{\frac{2 \pi i}{3} \sum_{n=1}^{|K|} k_{n}\right\} .
$$

Then we cut every sheet $\mathbf{C}_{K}$ along the three rays starting from $w_{K}^{j}$ and going in the direction of $\arg w_{K}^{j}$ to infinity, to obtain stars $H_{K}$. Thus $H_{K}$ is a plane with three radial cuts. We call these cuts $L_{K}^{j}, j \in\{0,1,2\}$. Notice that if $K^{\prime}$ is obtained from $K$ by adding on the right one digit $q \in\{1,2\}$, then the ray $L_{K}^{q}$ matches the ray $L_{K^{\prime}}^{0}$. Now we paste each such $H_{K^{\prime}}$ to $H_{K}$ along their matching cut in the usual way, respecting the projection, so that the starting points of the cuts become simple critical points of the projection map $g$. To this we add the sheet $H_{0}$ which has three cuts $L_{00}, L_{01}$ and $L_{02}$. The last two cuts are made to match $L_{1}^{0}$ and $L_{2}^{0}$ respectively, so we paste $H_{1}$ and $H_{2}$ to $H_{0}$ along them. One cut, $L_{00}$ remains free. It is easy to check that in this way we obtain a simply connected bordered surface which we call $X^{\prime}$. The border consists of two sides of the remaining cut $L_{00}$. The projection map is topologically holomorphic in the interior of $X^{\prime}$. To remove the border we take another copy $X^{\prime \prime}$ of $X^{\prime}$ and paste it to $X^{\prime}$ along the free cut.

The resulting open surface is called $X$ and the projection map (which we still denote by the same letter) $g: X \rightarrow \mathbf{C}$ is topologically holomorphic. It follows from Lemma 6.1 that there is no hemisphere where an inverse branch exists. Indeed, no branch of the inverse $g^{-1}$ exists on any region containing any of the three intervals $l_{j}$ from Lemma 6.1. It remains to prove that the sequence $\rho_{k}$ can be chosen in such a way that the constructed Riemann surface $X$ has parabolic type. This can be seen using the Caratheodory Kernel Theorem [22]. But in fact there is even a quantitative result [23, Theorem 50]: if we assume $1<\rho_{1} / \rho_{2} \leq \rho_{2} / \rho_{3} \leq \ldots$ then

$$
\sum_{k=1}^{\infty} \frac{1}{2^{n}} \log \log \frac{\rho_{n}}{\rho_{n+1}}=\infty
$$

is necessary and sufficient for parabolic type of $X$.

\section{Facts from spherical geometry}

Unless otherwise stated, from now on all metric concepts will refer to the spherical metric. We denote by $\bar{B}(a, r)$ the closed spherical disc of radius $r>0$ centered at $a \in \overline{\mathbf{C}}$. Note that $\bar{B}(a, \pi)=\overline{\mathbf{C}}$, and that $\bar{B}(a, \pi / 2)$ is a closed hemisphere for $a \in \overline{\mathbf{C}}$. 
If $x, y \in \overline{\mathbf{C}}$ are two points which are not antipodal, we denote by $[x, y]$ the unique geodesic joining $x$ and $y$. The geodesic $[x, y]$ is the shorter arc of the two subarcs with endpoints $x$ and $y$ on the great circle which passes through $x$ and $y$.

A set $C$ is called spherically convex if it is contained in an open hemisphere and $[x, y] \subseteq C$ for all $x, y \in C$. According to this definition a closed hemisphere is not spherically convex. An intersection of a spherically convex set with a closed hemisphere is spherically convex.

A convex spherical cell $C$ is a compact spherically convex set with nonempty interior whose boundary is a finite union of (maximal) great circular arcs. These arcs are called the edges of $C$, and the endpoints of these arcs the vertices of $C$. Each cell has at least three vertices and edges. A spherical cell is the spherical convex hull of its vertices, i.e., the smallest spherically convex set containing the vertices.

Lemma 7.1 Suppose $\bar{B}_{1}$ and $\bar{B}_{2}$ are two closed discs in $\overline{\mathbf{C}}$ strictly smaller than hemispheres. For $j \in\{1,2\}$ let $C_{j}$ be a spherical cell contained in $\bar{B}_{j}$ whose set of vertices $V_{j}$ is contained in the boundary of $\bar{B}_{j}$. If $\bar{B}_{1} \neq \bar{B}_{2}$ and no point of $V_{1} \cup V_{2}$ lies in the interior of $\bar{B}_{1}$ or in the interior of $\bar{B}_{2}$, then one of the following possibilities occurs

(i) $C_{1} \cap C_{2}=\emptyset$,

(ii) $C_{1} \cap C_{2}$ is a common edge of $C_{1}$ and $C_{2}$,

(iii) $C_{1} \cap C_{2}$ is a common vertex of $C_{1}$ and $C_{2}$.

Proof. Suppose $C_{1} \cap C_{2} \neq \emptyset$. Then $\bar{B}_{1} \cap \bar{B}_{2} \neq \emptyset$. We have neither $\bar{B}_{1} \subseteq \bar{B}_{2}$ nor $\bar{B}_{1} \subseteq \bar{B}_{2}$. For $\bar{B}_{1}=\bar{B}_{2}$ is excluded by assumption. Strict inclusion of the discs $\bar{B}_{1}$ and $\bar{B}_{2}, \bar{B}_{1} \subset \bar{B}_{2}$ say, is also impossible, for in this case the interior of $\bar{B}_{2}$ would contain at least two of the vertices of $C_{1}$. This is impossible by our hypotheses.

Hence, the discs $\bar{B}_{1}$ and $\bar{B}_{2}$ have nonempty intersection, but neither of the discs is contained in the other. Since both discs are strictly smaller than hemispheres, the following two possibilities may occur.

The intersection $\bar{B}_{1} \cap \bar{B}_{2}$ consists of a point which is then on the boundary of the discs. We have $\emptyset \neq C_{1} \cap C_{2} \subseteq \bar{B}_{1} \cap \bar{B}_{2}$. The vertices of $C_{1}$ and $C_{2}$ are the only points of $C_{1}$ and $C_{2}$ that lie on the boundary of $\bar{B}_{1}$ and $\bar{B}_{2}$, respectively. Hence $C_{1} \cap C_{2}$ consists of a common vertex of $C_{1}$ and $C_{2}$, and we are in case (iii).

The intersection $\bar{B}_{1} \cap \bar{B}_{2}$ contains more than one point. Then it is the closure of a lens-shaped region bounded by two nondegenerate $\operatorname{arcs} \alpha_{1} \subseteq$ 
$\partial \bar{B}_{1}$ and $\alpha_{2} \subseteq \partial \bar{B}_{2}$ with common endpoints $x, y, x \neq y$, where $\{x, y\}=$ $\partial \bar{B}_{1} \cap \partial \bar{B}_{2}$. The open $\operatorname{arcs} \beta_{1}=\alpha_{1} \backslash\{x, y\}$ and $\beta_{2}=\alpha_{2} \backslash\{x, y\}$ lie in the interior of $\bar{B}_{2}$ and $\bar{B}_{1}$, respectively. This implies $\beta_{1} \cap V_{1}=\emptyset$ and $\beta_{2} \cap V_{2}=\emptyset$. Let $H_{1}$ and $H_{2}$ be the two closed hemispheres determined by the great circle containing $[x, y]$. One of them contains $\alpha_{1}$ and the other one contains $\alpha_{2}$. We use notation so that $\alpha_{2} \subseteq H_{1}$ and $\alpha_{1} \subseteq H_{2}$. Then $V_{1} \subseteq \partial \bar{B}_{1} \backslash \beta_{1} \subseteq H_{1}$. This implies that the spherical convex hull of $V_{1}$ which is $C_{1}$, is also contained in $H_{1}$. Similarly, it can be shown that $C_{2} \subseteq H_{2}$. Therefore, $C_{1} \cap C_{2} \subseteq \bar{B}_{1} \cap \bar{B}_{2} \cap H_{1} \cap H_{2}=[x, y]$. If $C_{1} \cap C_{2}=\{x\}$ or $C_{1} \cap C_{2}=\{y\}$, then we are again in case (iii). But if $C_{1} \cap C_{2} \subseteq[x, y]$ contains at least two points, then it must contain an interior point of the arc $[x, y]$. Since $C_{1}$ is the convex spherical hull of $V_{1} \subseteq\left(\partial \bar{B}_{1} \backslash \beta_{1}\right)$, and $C_{2}$ is the hull of $V_{2} \subseteq\left(\partial \bar{B}_{2} \backslash \beta_{2}\right)$, this is only possible if $x, y \in V_{1} \cap V_{2}$. Then $C_{1} \cap C_{2}=[x, y]$ and $[x, y]$ is an edge of both $C_{1}$ and $C_{2}$. Hence we have case (ii).

If $E \subseteq \overline{\mathbf{C}}$ is a Borel set, we denote by $|E|$ its spherical area. We need the following elementary geometric fact. We outline a proof for the sake of completeness.

Lemma 7.2 Among all spherical triangles contained in a closed disc $\bar{B}$ of radius $R<\pi / 2$, equilateral triangles with vertices on $\partial B$ have the largest spherical area. If the maximal area is $A=\pi \delta$, then $R=b(\delta)$, where $b$ is defined as in (3).

Proof. For $0<t<\pi / 2$ let $S(t)$ be the spherical area of the equilateral triangle contained in a closed disc of radius $t$ and whose vertices lie on the boundary of the disc, i.e., whose circumscribed radius is $t$. Obviously, $S(t)$ is non-decreasing in $t$. We have to show that if a triangle $\Delta$ is contained in $\bar{B}$, then $|\Delta| \leq S(R)$. Since $\Delta \subseteq \bar{B}$, we have $r \leq R$, where $r$ is the circumscribed radius of $\Delta$. Let $r_{0}$ be the circumscribed radius of the equilateral triangle with the same spherical area as $\Delta$. If we can show $r_{0} \leq r$, then $|\Delta|=S\left(r_{0}\right) \leq S(r) \leq S(R)$, and we are done.

To prove $r_{0} \leq r$, it suffices to show that among all triangles with fixed spherical area strictly less than $2 \pi$ (the hemisphere area), the equilateral one has the smallest circumscribed radius. If $\alpha, \beta, \gamma$ are the angles of a spherical triangle, then its area is $|\Delta|=\alpha+\beta+\gamma-\pi[6$, p. 229, 127]. Fixing the area as a value in $(0,2 \pi)$ amounts to the same as fixing $\sigma=$ $\frac{1}{2}(\alpha+\beta+\gamma) \in(\pi / 2,3 \pi / 2)$. 
The circumscribed radius of $\Delta$ is given by

$$
\cot r=\sqrt{-\frac{\cos (\sigma-\alpha) \cos (\sigma-\beta) \cos (\sigma-\gamma)}{\cos (\sigma)}}
$$

(see, for example [6, p. 247, (310)]). We have to prove that under the additional restriction $0 \leq \alpha, \beta, \gamma \leq \pi$ the value of $r$ for given $\sigma \in(\pi / 2,3 \pi / 2)$ becomes minimal for $\alpha=\beta=\gamma$, i.e., in the equilateral case.

Formula (16) shows that we have to make

$$
2 \cos (\sigma-\alpha) \cos (\sigma-\beta) \cos (\sigma-\gamma)=(\cos (\gamma)+\cos (\alpha-\beta)) \cos (\sigma-\gamma)
$$

maximal under the given restrictions. By compactness of the allowed range for the angles and the continuity of the function to maximize, the maximum is attained, for $\alpha_{0}, \beta_{0}, \gamma_{0}$ say. The maximum is positive as the equilateral case shows. So for the maximum all the cosine terms of the left hand side of (17) are positive or one is positive and two are negative. Note that $-\pi / 2<\sigma-\pi \leq \sigma-\alpha \leq \sigma<3 \pi / 2$. Similar inequalities are true for $\sigma-\beta$ and $\sigma-\gamma$. So if two cosine terms are negative, the first two say, then $\sigma-\alpha_{0}>\pi / 2$ and $\sigma-\beta_{0}>\pi / 2$. Hence $\gamma_{0}=2 \sigma-\alpha_{0}-\beta_{0}>\pi$ which is impossible. Therefore, for the maximum all cosine terms on the left hand side of (17) are positive.

Now suppose two of the angles $\alpha_{0}, \beta_{0}, \gamma_{0}$ differ from each other, $\alpha_{0} \neq \beta_{0}$, say. Since $\cos \left(\sigma-\gamma_{0}\right)>0$, the right hand side of (17) shows that we can make the expression strictly larger by keeping $\gamma=\gamma_{0}$ and replacing $\alpha_{0}$ and $\beta_{0}$ by their mean value. This is a contradiction. Hence we must have $\alpha_{0}=\beta_{0}=\gamma_{0}$.

\section{Tilings of Riemann surfaces by triangles}

Suppose $f: X \rightarrow \overline{\mathbf{C}}$ is a non-constant meromorphic function on a Riemann surface $X$. Recall that $\mu_{f}(p)$ stands for the local degree of $f$ at a point $p \in X$. The set of critical points is denoted by $R_{f}$.

If $X$ is compact, $\operatorname{deg} f$ is the degree of $f$ and $g(X)$ the genus of $X$, then the Riemann-Hurwitz formula implies

$$
\sum_{p \in R_{f}}\left(\mu_{f}(p)-1\right)=2 \operatorname{deg} f+2 g(X)-2 .
$$

In the following lemma we consider an open Riemann surface $X$ and equip it with a complete Riemannian metric as discussed in Section 2. All 
metric notions on $X$ will refer to this fixed metric. The metric space $X$ is proper, i.e., closed balls are compact. In the following proof, this is important for the application of Ascoli's Theorem.

Lemma 8.1 Let $X$ be an open Riemann surface, and $f: X \rightarrow \overline{\mathbf{C}}$ a nonconstant holomorphic map. Assume for $k \in \mathbf{N}$ that $\gamma_{k}$ is a rectifiable curve in $X$ with initial point $x_{k} \in X$ and endpoint $y_{k} \in X$ for $k \in \mathbf{N}$. If $\left(x_{k}\right)$ is a bounded sequence, $\left(y_{k}\right) \rightarrow \infty$ for $k \rightarrow \infty$, and the spherical length of $f \circ \gamma_{k}$ is bounded by a finite constant independent of $k$, then $f$ has an asymptotic curve.

Proof. Fix a point $q \in X$ and let $p_{k}, k \in \mathbf{N}$, be an enumeration of the critical points of $f$. We can choose small numbers $\delta_{k}$ with the following properties. The closed discs $\bar{D}\left(p_{k}, \delta_{k}\right), k \in \mathbf{N}$, are pairwise disjoint, $\sup _{k \in \mathbf{N}} \delta_{k} \leq 1$, and if $\epsilon_{k}$ is the spherical length of the image of the circle $\partial D\left(p_{k}, \delta_{k}\right)$ under $f$, then $\sum_{k=1}^{\infty} \epsilon_{k} \leq 1$.

For any rectifiable curve $\gamma \subseteq X$, there is a rectifiable curve $\tilde{\gamma} \subseteq X$ such that the initial points of $\gamma$ and $\tilde{\gamma}$, and the endpoints of these curves are within distance 1 , for the spherical lengths of the curves under the map $f$ we have $\operatorname{length}_{s}(f \circ \tilde{\gamma}) \leq \operatorname{length}_{s}(f \circ \gamma)+1$, and $\tilde{\gamma}$ has empty intersection with $M=\bigcup_{k \in \mathbf{N}} D\left(p_{k}, \delta_{k}\right)$.

The curve $\tilde{\gamma}$ is inductively constructed as follows. Assume the initial point and the end point of $\gamma$ are not in $D\left(p_{1}, \delta_{1}\right)$. If $\gamma$ hits $D\left(p_{1}, \delta_{1}\right)$ at all, there is a first and a last point on the curve belonging to $\bar{D}\left(p_{1}, \delta_{1}\right)$. Replace the curve between these two points by an arc on $\partial D\left(p_{1}, \delta_{1}\right)$ connecting these points. This will increase the spherical length of the image under $f$ by at most $\epsilon_{1}$. A slight modification of this construction is necessary, if the initial point or the endpoint of $\gamma$ happens to be in $\bar{D}\left(p_{1}, \delta_{1}\right)$. Proceed like this also with the other discs $\bar{D}\left(p_{k}, \delta_{k}\right)$. This is a finite process, since $\gamma$ meets only finitely many of them.

Replacing our given curves $\gamma_{k}$ by these modified curves, we may in addition to our hypotheses assume that none of them meets the set $M$.

Assume that the curves $\gamma_{k}:\left[0, L_{k}\right] \rightarrow X$ are given in arclength parametrization with respect to our metric on $X$ such that $\gamma_{k}(0)=x_{k}$. Then we obviously have $\lim _{k \rightarrow \infty} L_{k}=\infty$. Using Ascoli's theorem and passing to an appropriate subsequence if necessary, we may furthermore assume that the parametrizations of our curves converge compactly on $[0, \infty)$ to the parametrization of a curve $\gamma:[0, \infty) \rightarrow X$. The curve $\gamma$ then does not meet $M$ and the spherical length of the image of $\gamma$ under $f$ is finite, since the spherical length of the image of $\gamma_{k}$ is bounded above by a constant independent of $k$. Hence $\lim _{t \rightarrow \infty} f \circ \gamma(t)$ exists. 
If we can show that $\lim _{t \rightarrow \infty} \gamma(t)=\infty$ (here $\infty$ is of course the "infinite point" of the one-point compactification of $X$ ), then $\gamma$ is an asymptotic curve.

The spherical derivative of $f$ is the ratio of the spherical length element on $\overline{\mathbf{C}}$ pulled back to $X$ by $f$ and the length element on $X$. Note that both length elements are in the same conformal class, so this makes sense. The spherical derivative is a continuous function on $X$ vanishing exactly at the branch points of $f$. Let $n \in \mathbf{N}$ be arbitrary. The spherical derivative on $\bar{D}(q, n) \backslash M$ is bounded from below by a positive constant only depending on $n$. Hence the length of $\gamma_{k} \cap \bar{D}(q, n)$ is less than a constant $l_{n}$ independent of $k$. It follows that for $k$ sufficiently large, there exits $t_{k} \in\left[0, l_{n}\right]$ such that $\gamma_{k}\left(t_{k}\right) \notin D(q, n)$. Considering the limit of a subsequence of $\left(t_{k}\right)$ and using uniform convergence of the parametrizations of $\gamma_{k}$ on $\left[0, l_{n}\right]$, we see that there exists $s_{n} \in\left[0, l_{n}\right]$ such that $\gamma\left(s_{n}\right) \notin D(q, n)$. In this way we obtain a sequence $\left(s_{n}\right)$ such that $\lim _{n \rightarrow \infty} \gamma\left(s_{n}\right)=\infty$.

Now it is clear that $\gamma$ ultimately leaves any disc $D(q, R), R>0$, and hence any compact subset of $X$. For by what we have seen, $\gamma$ will leave $D(q, 2 R)$ after each visit to $D(q, R)$. For each such visit, the spherical length of the image of $\gamma$ under $f$ will pick up a fixed positive amount. Since the spherical length of $\gamma$ is finite, this can only happen finitely often.

For a fixed meromorphic function $f$ on a Riemann surface $X$ recall the definition of $d_{f}(p)$ given in the Introduction. The function $d_{f}(p)$ is continuous in $p$. If $p \in X \backslash R_{f}$, then a single-valued branch of $f^{-1}$ inverting $f$ at $p$ can be defined in the disc $B_{f}(p):=B\left(f(p), d_{f}(p)\right)$. We denote it by $\Phi_{p}$. It is the unique holomorphic map $\Phi_{p}: B_{f}(p) \rightarrow X$ such that $\Phi_{p}(f(p))=p$ and $f \circ \Phi_{p}=\operatorname{id}_{B_{f}(p)}$.

Lemma 8.2 Let $X$ be a Riemann surface and $f: X \rightarrow \overline{\mathbf{C}}$ a non-constant holomorphic map without asymptotic values.

Then for $p \in X \backslash R_{f}$, the local inverse map $\Phi_{p}$ defined in $B_{f}(p)$ as above, has a continuous extension as a map of $\bar{B}_{f}(p)$ into $X$ (also denoted by $\Phi_{p}$ ). The function $\Phi_{p}$ is injective on $\bar{B}_{f}(p)$, and we have $f \circ \Phi_{p}=\operatorname{id}_{\bar{B}_{f}(p)}$.

If $q \in \partial B_{f}(p)$ is a singular point of $\Phi_{p}$, then $\Phi_{p}(q) \in R_{f}$. Such a point $q$ always exists, unless $X=\overline{\mathbf{C}}$ and $f: \overline{\mathbf{C}} \rightarrow \overline{\mathbf{C}}$ is an automorphism (i.e., fractional-linear).

This follows from Iversen's theorem, formulated in Section 3. We omit the details. 
Lemma 8.3 Let $X$ be a Riemann surface and $f: X \rightarrow \overline{\mathbf{C}}$ a non-constant holomorphic map without asymptotic values such that $d_{f}(p)<\pi / 2$ for all $p \in X$.

(a) Suppose $p_{1} \in X \backslash R_{f}$, and $x, y \in \partial B_{f}\left(p_{1}\right), x \neq y$, are singularities of $\Phi_{p_{1}}$. Let $\alpha$ be one of the open subarcs of $\partial B_{f}\left(p_{1}\right)$ with endpoints $x$ and $y$, and assume its interior does not contain singularities of $\Phi_{p_{1}}$. Then there exists a point $p_{2} \in X \backslash R_{f}$ such that $\bar{B}_{f}\left(p_{1}\right) \neq \bar{B}_{f}\left(p_{2}\right)$, $B_{f}\left(p_{1}\right) \cap B_{f}\left(p_{2}\right) \neq \emptyset, x, y \in \partial B_{f}\left(p_{2}\right), \Phi_{p_{1}}=\Phi_{p_{2}}$ on $B_{f}\left(p_{1}\right) \cap B_{f}\left(p_{2}\right)$, and $\Phi_{p_{2}}$ has a singularity on $\partial B_{f}\left(p_{2}\right)$ different from $x$ and $y$.

(b) There exists a point $p \in X \backslash R_{f}$ such that $\partial B_{f}(p)$ contains at least three singularities of $\Phi_{p}$.

Proof. (a) Let $H$ be the open hemisphere bounded by the great circle passing through $x$ and $y$ which contains $\alpha$. As the singularities $x$ and $y$ of $\Phi_{p_{1}}$ come from critical points of $f$, the function $\Phi_{p_{1}}$ has direct analytic continuation to $\alpha_{\epsilon} \cap H$, where $\alpha_{\epsilon}$ is the spherical $\epsilon$-neighborhood of $\alpha$. Let $m$ be the midpoint of $\alpha$, and $\gamma:[0,2 \pi] \rightarrow \overline{\mathbf{C}}$ an arclength parametrization of the great circle containing $\left[p_{1}, m\right]$ such that $\gamma(0)=p_{1}$ and $\gamma(t) \in\left[p_{1}, m\right]$ for small values of $t$. Let $t_{0} \in[0, \pi]$ be the supremum of all values for which the analytic continuation $\Phi$ of $\Phi_{p_{1}}$ along $\gamma \mid\left[0, t_{0}\right)$ exists. Obviously, $t_{0}>0$. Iversen's Theorem shows that the function $\Phi \circ \gamma \mid\left[0, t_{0}\right)$ has a continuous extension to $\left[0, t_{0}\right]$.

Both $t \mapsto d_{1}(t):=d_{f}(\Phi(\gamma(t)))$ and $t \mapsto d_{2}(t):=\operatorname{dist}(\gamma(t),\{x, y\})$ are continuous functions on $\left[0, t_{0}\right]$. The existence of the direct analytic continuation of $\Phi_{p_{1}}$ to $\alpha_{\epsilon} \cap H$ implies that $d_{1}(t)=d_{2}(t)$ for small $t \geq 0$. Now if $t_{0}<\pi$, then $\Phi\left(\gamma\left(t_{0}\right)\right)$ is a critical point of $f$ and so $d_{1}\left(t_{0}\right)=0$. Since $d_{2}$ is bounded away from zero, there exists a largest value $t_{1} \in\left(0, t_{0}\right)$ such that $d_{1}\left(t_{1}\right)=d_{2}\left(t_{1}\right)$.

If $t_{0}=\pi$, then $\gamma\left(t_{0}\right)$ is the center of the disc complementary to $B_{f}\left(p_{1}\right)$ and has radius larger than $\pi / 2$. Hence $d_{1}(\pi)=d_{f}(\Phi(\gamma(\pi)))<\pi / 2<d_{2}(\pi)$ by our hypotheses. Again there exists a largest value $t_{1} \in\left(0, t_{0}\right)$ such that $d_{1}\left(t_{1}\right)=d_{2}\left(t_{1}\right)$.

It is straightforward to show that $p_{2}=\Phi\left(\gamma\left(t_{1}\right)\right)$ has the required properties.

(b) Pick $p_{0} \in X \backslash R_{f}$ and let $q_{0}=f\left(p_{0}\right)$. Since $d_{f}(p)<\pi / 2$, there exists a singularity $x \in \partial B_{f}\left(p_{0}\right)$ of $\Phi_{p_{0}}$. For $q \in \overline{\mathbf{C}}$ let $B(q)$ be the open disc centered at $q$ which contains $x$ in its boundary. Starting at $q_{0}$, move a point $q$ on the great circle through $q_{0}$ and $x$ away from $x$ until for $q=q_{1}$ the analytic continuation $\Phi$ of $\Phi_{p_{0}}$ into $B(q)$ has a singularity $y \in \partial B(q)$ 
different from $x$ for the first time. Put $p_{1}=\Phi\left(q_{1}\right)$. Then $B_{f}\left(p_{1}\right)=B\left(q_{1}\right)$ and $x, y \in \partial B_{f}\left(p_{1}\right)$. If $\Phi_{p_{1}}$ has a singularity on $\partial B_{f}\left(p_{1}\right)$ different from $x$ and $y$, then $p=p_{1}$ has the required properties. If not, we repeat the argument in (a).

Proposition 8.4 Let $X$ be a Riemann surface, and $f: X \rightarrow \overline{\mathbf{C}}$ be a nonconstant holomorphic map without asymptotic values such that $d_{f}(p)<\pi / 2$ for all $p \in X$.

Then there exist exists a set $T$ of compact topological triangles in $X$ with the following properties

(a) For all $\Delta \in T$, the edges of $\Delta$ are analytic arcs, $f \mid \Delta$ is injective and conformal on $\Delta$. The set $\Delta^{\prime}=f(\Delta)$ is a spherical triangle contained in a closed spherical disc of radius $\mathfrak{B}_{f}$.

(b) If the intersection of two distinct triangles $\Delta_{1}, \Delta_{2} \in T$ is nonempty, then $\Delta_{1} \cap \Delta_{2}$ consists of exactly one common edge of $\Delta_{1}$ and $\Delta_{2}$ or is a set of common vertices.

(c) The set consisting of all the vertices of the triangles $\Delta \in T$ is equal to the set of critical points of $f$.

(d) $T$ is locally finite, i.e., for every $p \in X$ there exists a neighborhood $W$ of $p$ such that $W \cap \Delta \neq \emptyset$ for only finitely many $\Delta \in T$.

(e) $\bigcup_{\Delta \in T} \Delta=X$.

In general the triangles $\Delta \in T$ will not from a triangulation according to the usual definition, since two triangles can have two or three vertices in common without sharing an edge. For example, this situation may arise when two triangles lie above each other on different "sheets" of $X$ over $\overline{\mathbf{C}}$ but some of their vertices coincide.

As the proof below will show, for $\Delta \in T$ the spherical triangle $f(\Delta)$ is contained in a disc $\bar{B}_{f}(p)$ for some $p \in X$.

Proof of Proposition 8.4. We will first construct a set $\mathcal{K}$ of compact topological cells in $X$ with the following properties.

(a') For all $C \in \mathcal{K}$, the edges of $C$ are analytic arcs, $f \mid C$ is injective and conformal on $\stackrel{\circ}{C}$. The set $C^{\prime}=f(C)$ is a convex spherical cell contained in a closed spherical disc of radius $\mathfrak{B}_{f}$. 
(b') If the intersection of two distinct cells $C_{1}$ and $C_{2}$ is nonempty, then $C_{1} \cap C_{2}$ consists of exactly one common edge of $C_{1}$ and $C_{2}$, or is a set of common vertices.

$\left(c^{\prime}\right)$ Every vertex of a cell $C \in \mathcal{K}$ is a critical point of $f$.

$\left(d^{\prime}\right)$ The complex $\mathcal{K}$ does not contain free edges, i.e., every edge of a cell in $\mathcal{K}$ is the edge of exactly two cells in $\mathcal{K}$.

Define $\mathcal{C}$ to be the set of all $p \in X \backslash R_{f}$ such that the single valued branch $\Phi_{p}$ of $f^{-1}$ defined above has at least three singularities on $\partial B_{f}(p)$.

By Lemma 8.2 we have $\mathcal{C} \neq \emptyset$. Moreover, since $\Phi_{p}$ is injective on $\bar{B}_{f}(p)$ the image of $B_{f}(p)$ under $\Phi_{p}$ is a Jordan region $\Omega(p) \subseteq X$ compactly contained in $X$. The map $f$ restricted to $\overline{\Omega(p)}$ is a bijective map onto $\bar{B}_{f}(p)$ with the inverse map $\Phi_{p}$. The singularities of $\Phi_{p}$ on $\partial B_{f}(p)$ are mapped by $\Phi_{p}$ onto critical points of $f$ which lie on $\partial \Omega(p)$. Since the set of critical points of $f$ is discrete, there are only finitely many singularities of $\Phi_{p}$ on $\partial B_{f}(p)$.

Denote these singularities by $w_{0}, \ldots, w_{m}$. If $p \in \mathcal{C}$, then $m \geq 2$. Let $C^{\prime}(p)$ be the spherical convex hull of $w_{0}, \ldots, w_{m}$. Note that $C^{\prime}(p)$ is welldefined, because the points are contained in the disc $\bar{B}_{f}(p)$ which is strictly smaller than a hemisphere by our assumption $d_{f}(p)<\pi / 2$.

Let $C(p)=\Phi_{p}\left(C^{\prime}(p)\right)$. Then $C(p)$ is a topological cell whose vertices $\Phi_{p}\left(w_{0}\right), \ldots, \Phi_{p}\left(w_{m}\right)$ are critical points of $f$. We claim that $\mathcal{K}=\{C(p)$ : $p \in \mathcal{C}\} \neq \emptyset$ is a set of cells with the required properties.

Apart from the vertices, $C(p)$ lies in $\Omega(p)$ for $p \in \mathcal{C}$. Hence it does not contain any other critical points of $f$. In particular, the edges of $C(p)$ are analytic arcs. The map $f \mid C(p)$ is injective and conformal on $\stackrel{\circ}{C}(p)$, and $f(C(p))=C^{\prime}(p) \subseteq \bar{B}_{f}(p) \subseteq \bar{B}\left(f(p), \mathfrak{B}_{f}\right)$ is a convex spherical cell contained in a closed disc of radius $\mathfrak{B}_{f}$. Hence $\left(\mathrm{a}^{\prime}\right)$ and $\left(\mathrm{c}^{\prime}\right)$ are true.

To show that for two distinct cells $C_{1}=C\left(p_{1}\right)$ and $C_{2}=C\left(p_{2}\right), p_{1}, p_{2} \in$ $\mathcal{C}$ we have $\left(\mathrm{b}^{\prime}\right)$, we may assume that $C_{1}$ and $C_{2}$ have a point in common which is not a vertex.

Let $\bar{B}_{j}=\bar{B}_{f}\left(p_{j}\right), \Omega_{j}=\Omega\left(p_{j}\right), C_{j}^{\prime}=C^{\prime}\left(p_{j}\right)$, and $\Phi_{j}=\Phi_{p_{j}}$ for $j \in$ $\{1,2\}$. Then $\Omega_{1} \cap \Omega_{1} \neq \emptyset$. The uniqueness theorem for analytic functions implies $\Phi_{1}\left|\bar{B}_{1} \cap \bar{B}_{2}=\Phi_{2}\right| \bar{B}_{1} \cap \bar{B}_{2}$. Hence $C_{1} \cap C_{2}=\Phi_{1}\left(C_{1}^{\prime} \cap C_{2}^{\prime}\right)=\Phi_{2}\left(C_{1}^{\prime} \cap\right.$ $C_{2}^{\prime}$ ). This shows that $\bar{B}_{1} \neq \bar{B}_{2}$, for otherwise $C_{1}=C_{2}$, because the cells $C(p)$ for $p \in \mathcal{C}$ are uniquely determined by the branch $\Phi_{p}$ and the disc $\bar{B}_{f}(p)$. The assertion now follows from an application of Lemma 7.1 to the discs $\bar{B}_{1}, \bar{B}_{2}$ and the spherical cells $C_{1}^{\prime}, C_{2}^{\prime}$.

It remains to show $\left(\mathrm{d}^{\prime}\right)$. It is clear from plane topology that no edge can 
belong to three or more cells. So if $\gamma$ is an edge of a cell $C\left(p_{1}\right), p_{1} \in \mathcal{C}$, we have to show that there exists a cell in $\mathcal{K}$ distinct from $C\left(p_{1}\right)$ containing $\gamma$ as an edge.

Since $\gamma$ is an edge of $C\left(p_{1}\right)$, there exist singularities $x, y \in \partial B_{f}\left(p_{1}\right)$ of $\Phi_{p_{1}}$ such that $\gamma=\Phi_{p_{1}}([x, y])$. The points $x$ and $y$ are vertices of $C^{\prime}\left(p_{1}\right)$ and follow upon each other in any cyclic order of the vertices of $C_{1}^{\prime}$. Hence, there exists an open subarc $\alpha$ of $\partial B_{f}\left(p_{1}\right)$ with endpoints $x$ and $y$ not containing any singularities of $\Phi_{p_{1}}$. Let $p_{2}$ be the point constructed in Lemma 8.3 (a). Then $p_{2} \in \mathcal{C}$. Since $\bar{B}_{f}\left(p_{1}\right) \neq \bar{B}_{f}\left(p_{2}\right)$, we have $\{x, y\}=$ $\partial B_{f}\left(p_{1}\right) \cap \partial B_{f}\left(p_{2}\right)$. Both $\partial B_{f}\left(p_{1}\right)$ and $\partial B_{f}\left(p_{2}\right)$ contain a vertex of $C^{\prime}\left(p_{1}\right)$ and $C^{\prime}\left(p_{2}\right)$, respectively, different from $x$ and $y$. Since those vertices lie on $\partial B_{f}\left(p_{1}\right)$ and $\partial B_{f}\left(p_{2}\right)$, respectively, and $\{x, y\}=\partial B_{f}\left(p_{1}\right) \cap \partial B_{f}\left(p_{2}\right)$, the sets $C^{\prime}\left(p_{1}\right)$ and $C^{\prime}\left(p_{2}\right)$ are different. Hence $C\left(p_{1}\right) \neq C\left(p_{2}\right)$. One of the open subarcs of $\partial B_{f}\left(p_{2}\right)$ with endpoints $x$ and $y$ lies in $B_{f}\left(p_{1}\right)$. Hence it contains no singularities of $\Phi_{p_{2}}$. In particular, $x$ and $y$ follow upon each other in any cyclic order of the singularities of $\Phi_{p_{2}}$ on $\partial B_{f}\left(p_{2}\right)$. Hence $[x, y]$ is an edge of $C^{\prime}\left(p_{2}\right)$. This shows that $\gamma=\Phi_{p_{1}}([x, y])=\Phi_{p_{2}}([x, y])$ is a common edge of $C\left(p_{1}\right)$ and $C\left(p_{2}\right)$.

We have established $\left(\mathrm{a}^{\prime}\right)-\left(\mathrm{d}^{\prime}\right)$. The set $T$ is now constructed as follows. For $p \in \mathcal{C}$ let $w_{0}, w_{1}, \ldots, w_{m}, m \geq 2$, be the vertices of $C^{\prime}(p)$ arranged in cyclic order on $\partial C^{\prime}(p)$, which is a topological circle. For $j \in$ $\{1, \ldots, m-1\}$ let $\Delta_{j}^{\prime}$ be the spherical triangle with the vertices $w_{0}, w_{j}, w_{j+1}$ contained in $C^{\prime}(p)$, and let $\Delta_{j}=\Phi_{p}\left(\Delta_{j}^{\prime}\right)$. Then the toplogical triangles $\Delta_{1}, \Delta_{2}, \ldots, \Delta_{m-1}$ form a triangulation of $C(p)$. Let $T$ be the set of all the triangles thus obtained by triangulating the cells in $\mathcal{K}$. The properties (a) and (b) follow from the corresponding properties of $\mathcal{K}$. Moreover, it is clear that $T$ does not contain free edges, and that every vertex of a triangle in $T$ is a critical point.

To show that $T$ is locally finite we first show that for three distinct points $p_{1}, p_{2}, p_{3} \in R_{f}$ there are only finitely many triangles in $T$ having these points as vertices. So suppose the triangles $\Delta_{1}, \ldots, \Delta_{l} \in T$ all have the vertices $p_{1}, p_{2}, p_{3}$. Then the spherical triangles $\Delta_{j}^{\prime}=f\left(\Delta_{j}\right)$ coincide for $j \in\{1, \ldots, l\}$, since they all are identical to the convex hull $\Delta$ of $q_{1}=f\left(p_{1}\right)$, $q_{2}=f\left(p_{2}\right), q_{3}=f\left(p_{3}\right)$. We claim that $l \leq \min \left\{\mu_{f}\left(p_{1}\right), \mu_{f}\left(p_{2}\right), \mu_{f}\left(p_{3}\right)\right\}$. Assume the minimum is given by $\mu_{f}\left(p_{1}\right)$, say. Since the function $f$ restricted to $\Delta_{j}$ gives a bijective map of $\Delta_{j}$ onto $\Delta$ which is conformal in the interior of $\Delta_{j}$, each point $q$ in the interior of $\Delta$ close to $q_{1}$ will have a preimage under $f$ in the interior of $\Delta_{j}$ close to $p_{1}$. Since the interiors of the triangles $\Delta_{j}$ do not overlap, it follows that in this way we get $l$ preimages of $q$ close to $p_{1}$. Hence $l \leq \mu_{f}\left(p_{1}\right)$. 
Now suppose that $T$ is not locally finite. If $X$ is compact, then $R_{f}$ and so $T$ is a finite set by what we have just seen. Hence $X$ is open. There exists a point $p \in X$ and a compact neighborhood $W$ of $p$ which meets infinitely many distinct triangles $\Delta_{k} \in T, k \in \mathbf{N}$. Pick a point $x_{k} \in \Delta_{k} \cap W$ for $k \in \mathbf{N}$.

The set $V$ of all the vertices of the triangles $\Delta_{k}$ is infinite, for by what we have seen above, for each three element subset $A$ of $R_{f}$ there are only a finite number of distinct triangles in $T$ having $A$ as its set of vertices. Since $R_{f}$ is discrete in $X$, the set $V$ has the point at infinity as an accumulation point. Passing to a subsequence of the triangles $\Delta_{k}$ if necessary, it follows that we may assume that for each $k \in \mathbf{N}$ we can choose a vertex $y_{k}$ of the triangle $\Delta_{k}$ such that $\left(y_{k}\right) \rightarrow \infty$. The restriction $f \mid \Delta_{k}$ is injective, and maps $\Delta_{k}$ onto a spherical triangle $\Delta_{k}^{\prime}$ contained in a disc of radius $\mathfrak{B}_{f}$, and hence in a hemisphere. For $k \in \mathbf{N}$ connect the points $x_{k}^{\prime}=f\left(x_{k}\right)$ and $y_{k}^{\prime}=f\left(y_{k}\right)$ by the geodesic $\gamma^{\prime}=\left[x_{k}^{\prime}, y_{k}^{\prime}\right] \subseteq \Delta_{k}^{\prime}$. Let $\gamma_{k}$ be the inverse image of $\gamma_{k}^{\prime}$ under $f \mid \Delta_{k}$. Then for $k \in \mathbf{N}$, the curve $\gamma_{k}$ is a locally rectifiable curve in $X$ with endpoints $x_{k}$ and $y_{k}$. The image of $\gamma_{k}$ under $f$ is equal to $\gamma_{k}^{\prime}$. Hence the spherical length of the image curve of $\gamma_{k}$ under $f$ does not exceed $\pi$. Lemma 8.1 now implies that $f$ has an asymptotic value. This is a contradiction showing that $T$ is locally finite.

We now show that $U=\bigcup_{\Delta \in T} \Delta=X$. Since a locally finite union of closed sets is closed, $U$ is closed. Moreover, $U \neq \emptyset$, since $T \neq \emptyset$. Hence $U=X$ will follow from the connectedness of $X$ if we can show that $U$ is open. So we have to prove that $U$ only contains interior points.

If $z \in U$ is an interior point of a triangle $\Delta \in T$, then it is clearly an interior point of $U$. If $z$ is an interior point of an edge, then, since $T$ has no free edges, the edge belongs to two distinct triangles $\Delta_{1}, \Delta_{2} \in T$. Then $\Delta_{1} \cup \Delta_{2} \subseteq U$ is a neighborhood of $z$. The remaining possibility is that $z$ is a vertex. Each triangle with vertex $z$ has exactly two edges containing $z$. Since there are no free edges in $T$, and $T$ is locally finite, the triangles of which $z$ is a vertex, can be arranged in a finite number of cycles such that two triangles following upon each other in a cycle share a common edge whose one endpoint is $z$. Plane topology shows that there exists only one such cycle containing $\Delta_{1}, \ldots, \Delta_{l} \in T$, say. Then $\Delta_{1} \cup \ldots \cup \Delta_{l} \subseteq U$ is a neighborhood of $z$.

It remains to prove the other half of (c). Namely, that every critical point $p$ is the vertex of a triangle in $T$. This is clear, since by (e) the point $p$ must be contained in some triangle $\Delta \in T$, and by construction of the triangles, no point of a triangle other than a vertex can be a critical point. 


\section{Proof of Theorem $\mathbf{1 . 5}$}

A reader not familiar with Nevanlinna theory is advised to read Section 10 first. The proofs of Theorems 1.5 and 1.7 are based on the same idea but in the case of algebraic functions no hard analysis is necessary.

All $o$ and $O$ symbols in this section refer to $r \rightarrow \infty$. Let

$$
f^{\#}:=\frac{2\left|f^{\prime}\right|}{1+|f|^{2}}
$$

be the spherical derivative ${ }^{3}$ of a meromorphic function $f: \mathbf{C} \rightarrow \overline{\mathbf{C}}$. The non-integrated Ahlfors characteristic is

$$
A(r):=\frac{1}{4 \pi} \int_{|z| \leq r}\left(f^{\#}\right)^{2}(z) d m_{2}(z),
$$

where $d m_{2}$ stands for integration with respect to Euclidean area. Nevanlinna's characteristic can be defined by

$$
T(r)=\int_{0}^{r} A(t) \frac{d t}{t} .
$$

Let $n_{1}(r)$ be the number of critical points of $f$ in $|z| \leq r$ counted according to multiplicity, and

$$
N_{1}(r)=\int_{0}^{r} \frac{n_{1}(t)-n_{1}(0)}{t} d t+n_{1}(0) \log r
$$

the corresponding averaged counting function.

A meromorphic function $f$ is normal if and only if $f^{\#}$ is bounded in C. Yosida [25] proved that a normal function belongs to the class $\mathcal{Y}$ if and only if the following additional condition is satisfied: for every $\delta>0$ there exists a constant $c_{1}=c_{1}(f, \delta)>0$ such that

$$
\int_{\left|z-z_{0}\right| \leq \delta}\left(f^{\#}\right)^{2}(z) d m_{2}(z)>c_{1} \quad \text { for every } \quad z_{0} \in \mathbf{C} .
$$

It follows that for $f \in \mathcal{Y}$ there exist positive constants $c_{2}$ and $c_{3}$ such that

$$
c_{2} r^{2} \leq A(r) \leq c_{3} r^{2}, \quad r>1 .
$$

The following result can be found in [25], with a worse error term, but Yosida's proof contains an error (his Lemma 1 is incorrect), so we include a proof.

\footnotetext{
${ }^{3}$ In most texts on the subject the spherical derivative is defined without the factor 2 . That is why our formula for the Ahlfors characteristic looks somewhat unusual.
} 
Lemma 9.1 For $f \in \mathcal{Y}$ we have

$$
N_{1}(r)=2 T(r)+O(1) .
$$

Proof. Consider the function

$$
u:=\log f^{\#}=\log \left|f^{\prime}\right|-\log \left(1+|f|^{2}\right)+\log 2 .
$$

It is easy to see that $u$ is a difference of two subharmonic functions, and we have in the sense of distributions:

$$
\Delta u=2 \pi \sum \delta_{z_{k}}-\left(f^{\#}\right)^{2} d m_{2}
$$

where $\delta_{z_{k}}$ denotes the unit mass at $z_{k}$ and the sum is over all critical points $z_{k}$ repeated according to multiplicity. We integrate the signed measure $(2 \pi)^{-1} \Delta u$ over discs $\{z:|z| \leq t\}$, then from 0 to $r$ against $d t / t$, and apply Jensen's formula to obtain

$$
N_{1}(r)-2 T(r)=\frac{1}{2 \pi} \int_{-\pi}^{\pi} u\left(r e^{i \theta}\right) d \theta=\frac{1}{2 \pi r} \int_{|z|=r} u(z)|d z| .
$$

It remains to estimate the integral in the right hand side.

We claim that if the arc $\alpha$ is the intersection of a circle of radius greater than 1 with a disc of radius 1 , then

$$
\left|\int_{\alpha} \log f^{\#}(z)\right| d z|| \leq c_{4},
$$

where $c_{4}$ is a constant depending only on $f$. Indeed, otherwise we could find a sequence of discs $D_{j}=D\left(a_{j}, 1\right)$ and a sequence of arcs $\alpha_{j} \subseteq D_{j}$ such that

$$
\left|\int_{\alpha_{j}} \log f^{\#}(z)\right| d z|| \rightarrow \infty \quad \text { as } \quad j \rightarrow \infty .
$$

On the other hand, the functions $f\left(\cdot+a_{j}\right)$ form a normal family, so passing to a subsequence we can assume $f\left(\cdot+a_{j}\right) \rightarrow g$ locally uniformly, where $g$ is a meromorphic function, and $g \not \equiv$ const by the definition of $\mathcal{Y}$. Choosing a subsequence once more we may assume that $\alpha_{j}-a_{j} \rightarrow \alpha$, where $\alpha$ is an arc of a circle or a straight line segment. All this contradicts (22), so (21) holds.

It follows from (21) that the right side of (20) is bounded.

A Borel set $E \subseteq(0, \infty)$ has zero density if

$$
\lim _{r \rightarrow \infty} \frac{m_{1}(E \cap(0, r])}{r}=0 .
$$

Here $m_{1}$ is the Lebesgue measure on $\mathbf{R}$. 
Lemma 9.2 Let $f \in \mathcal{Y}$. For every $\delta>0$ and $r_{0}>0$ the set $E:=\{r>0$ : $\left.A\left(r+r_{0}\right)>(1+\delta) A(r)\right\}$ has zero density.

Proof. For every point $r^{\prime} \in E$ consider the segment $\left[r^{\prime}-r_{0}, r^{\prime}+r_{0}\right]$. These segments cover $E$, so one can select a subset of these segments, such that every point $E$ belongs to at most 2 segments [9, $\S 1$, Remark 1]. Fix arbitrary $r>0$ and let $r_{1}<r_{2}<\ldots<r_{n}$ be the centers of the selected segments that belong to $[0, r]$. The variation of $\log A$ on each selected segment is at least $\log (1+\delta)$, so the total variation of $\log A$ on the union of the selected segments whose centers lie in $[0, r]$ is at least $(n / 2) \log (1+\delta)$. Thus, as $\log A$ is increasing, and $A(r)=O\left(r^{2}\right)$,

$$
(n / 2) \log (1+\delta) \leq \log A\left(r+r_{0}\right) \leq 2 \log r+O(1) .
$$

So we obtain $n=o(r), r \rightarrow \infty$. But the total length of the selected segments whose centers belong to $[0, r]$ is at most $2 n r_{0}$, from which the desired result follows.

We conclude from Lemma 9.2 that for every $r_{0}>0$ there is a set $E_{1}$ of zero density, such that

$$
A\left(r+r_{0}\right)=(1+o(1)) A(r), \quad r \notin E_{1} .
$$

Lemma 9.3 For $f \in \mathcal{Y}$ we have

$$
n_{1}(r) \leq(2+o(1)) A(r), \quad r \notin E_{1} .
$$

Proof. We apply Lemma 9.1:

$$
\begin{aligned}
n_{1}(r) \log \left(\frac{r+r_{0}}{r}\right) & \leq \int_{r}^{r+r_{0}} n_{1}(t) \frac{d t}{t}=N_{1}\left(r+r_{0}\right)-N_{1}(r) \\
& =2\left(T\left(r+r_{0}\right)-T(r)\right)+O(1) \\
& =2 \int_{r}^{r+r_{0}} A(t) \frac{d t}{t}+O(1) \\
& \leq 2 A\left(r+r_{0}\right) \log \left(\frac{r+r_{0}}{r}\right)+O(1) .
\end{aligned}
$$

Dividing by $\log \left(\left(r+r_{0}\right) / r\right) \sim r_{0} / r$, and using (23) and (19), we obtain

$$
n_{1}(r) \leq 2 A\left(r+r_{0}\right)+O(r) \leq(2+o(1)) A(r) \quad \text { for } \quad r \notin E_{1} .
$$

Lemma 9.4 Let $f \in \mathcal{Y}$. Then there exists a constant $r_{0}(f)>0$ with the following property: If $\Gamma:[0,1] \rightarrow \mathbf{C}$ is a rectifiable curve, and

$$
\int_{\Gamma} f^{\#}(z)|d z| \leq 2 \pi
$$

then the Euclidean diameter of $\Gamma$ is at most $r_{0}(f)$. 
Proof. Assume $\Gamma:[0,1] \rightarrow \mathbf{C}$ is a rectifiable curve whose image curve under $f$ has spherical length at most $2 \pi$ and Euclidean diameter of $\Gamma$ exceeds some $n \in \mathbf{N}$. Considering an appropiate subcurve if necessary, we may assume that $|\Gamma(0)-\Gamma(1)|>n$. Now we can inductively construct points $t_{0}<t_{1}<\ldots<t_{n}$ in $[0,1]$ such that $\left|\Gamma\left(t_{k}\right)-\Gamma\left(t_{k-1}\right)\right|=1$ and $\Gamma\left(\left[t_{k-1}, t_{k}\right]\right) \subseteq$ $\bar{D}\left(\Gamma\left(t_{k-1}\right), 1\right)$ for $k \in\{1, \ldots, n\}$. To see this let $t_{0}=0$. If $t_{k-1}$ has been selected, let $t_{k} \in\left[t_{k-1}, 1\right]$ be the first point such that $\left|\Gamma\left(t_{k}\right)-\Gamma\left(t_{k-1}\right)\right|=1$. Such a point exists for $k \leq n$ by our assumption $|\Gamma(0)-\Gamma(1)|>n$.

One of the $n$ subcurves $\Gamma \mid\left[t_{k-1}, t_{k}\right]$ of $\Gamma$ must have an image curve under $f$ with spherical length at most $2 \pi / n$.

Now we assume that the assertion of the lemma does not hold. By what we have just seen we can then find a sequence of Euclidean discs $\bar{D}\left(a_{j}, 1\right)$, $a_{j} \in \mathbf{C}$, such that each disc contains a rectifiable curve $\Gamma_{j}$ connecting its center $a_{j}$ with the circumference $\partial D\left(a_{j}, 1\right)$, and

$$
\int_{\Gamma_{j}} f^{\#}(z)|d z| \rightarrow 0 \quad \text { as } \quad j \rightarrow \infty .
$$

The functions $g_{j}:=f\left(\cdot+a_{j}\right), j \in \mathbf{N}$, form a normal family by our assumption on $f$. By choosing an appropiate subsequence, we can assume that $\left(g_{j}\right)$ converges to a meromorphic function $g$ locally uniformly. The definition of the class $\mathcal{Y}$ implies $g \not \equiv$ const. This contradicts the fact that the spherical length of $g_{j} \circ\left(\Gamma_{j}-a_{j}\right)$ tends to zero.

Proof of Theorem 1.5. Let $f \in \mathcal{Y}$ and $\mathfrak{B}_{f}<b_{0}$. It follows from Lemma 9.4 that $f$ has no asymptotic values (this is due to Yosida [25]). Proposition 8.4 gives a tiling $T$ of the plane consisting of topological triangles, satisfying (a), (b), (c), (d), (e). Lemma 7.2 implies that the spherical area of the image of a triangle in $T$ under the map $f$ is at most $(1-\epsilon) \pi$, where $\epsilon>0$. Moreover, Lemma 9.4 implies that the Euclidean diameters of all these triangles are bounded by a constant $r_{0}:=r_{0}(f)$.

Let $\Delta \in T$, denote by $\alpha, \beta, \gamma$ the angles of the spherical triangle $\Delta^{\prime}=$ $f(\Delta)$, and by $S(\Delta)$ the sum of these angles. Then for the spherical area of $\Delta^{\prime}$ we get

$$
\left|\Delta^{\prime}\right|=\alpha+\beta+\gamma-\pi .
$$

Since all our triangles $\Delta^{\prime}$ have area at most $(1-\epsilon) \pi$, it follows

$$
\left|\Delta^{\prime}\right|=\alpha+\beta+\gamma-\pi \leq(1 / 2-\delta)(\alpha+\beta+\gamma)=(1 / 2-\delta) S(\Delta),
$$

where $\delta:=\epsilon /(4-2 \epsilon)$.

Suppose $v \in V:=R_{f}$ is a vertex of the tiling, and let $\Delta_{1}, \cdots, \Delta_{l}$ be the triangles of $T$ which have $v$ as a vertex. Denote by $S(v)$ the sum of the 
angles of spherical triangles $f\left(\Delta_{1}\right), \ldots, f\left(\Delta_{l}\right)$ at the vertex $f(v)$. Angles between curves meeting at $v$ are increased by the factor $\mu_{f}(v) \geq 2$ under the map $f$. Hence

$$
S(v)=2 \pi \mu_{f}(v) \leq 4 \pi\left(\mu_{f}(v)-1\right) \quad \text { for } \quad v \in V .
$$

If we define

$$
S(r)=\sum_{v \in V,|v| \leq r} S(v)
$$

it follows

$$
S(r) \leq 4 \pi n_{1}(r) \text { for } \quad r>0 .
$$

Lemma 9.3 and (23) imply that that exists a set $E$ of zero density such that

$$
n_{1}\left(r+r_{0}\right) \leq(2+o(1)) A\left(r+r_{0}\right) \leq(2+o(1)) A(r) \quad \text { for } \quad r>0, r \notin E .
$$

For $r>0$ let $T_{r}$ be the set of all triangles having non-empty intersection with the disc $\bar{D}(0, r)$. Since the diameter of the triangles is bounded by $r_{0}$, it follows that $|v| \leq r+r_{0}$ whenever $v$ is a vertex of a triangle in $T_{r}$. Using this, the properties of $T,(24),(25)$, and (26), we obtain for large $r, r \notin E$,

$$
\begin{aligned}
4 \pi A(r) & \leq \sum_{\Delta \in T_{r}}|\Delta| \leq(1 / 2-\delta) \sum_{\Delta \in T_{r}} S(\Delta) \\
& \leq(1 / 2-\delta) \sum_{v \in V,|v| \leq r+r_{0}} S(v)=(1 / 2-\delta) S\left(r+r_{0}\right) \\
& \leq 2 \pi(1-2 \delta) n_{1}\left(r+r_{0}\right) \leq 4 \pi(1-\delta) A(r) .
\end{aligned}
$$

This contradiction proves Theorem 1.5.

\section{Algebraic functions}

The proof of Theorem 1.7 is based on the same idea as that of Theorem 1.5 with the following modifications: we do not need Nevanlinna theory or distortion control like in Lemma 9.4 anymore, but use the Riemann-Hurwitz formula instead. This gives us a better estimate where genus and degree are taken into account.

Proof of Theorem 1.7. Let $f: X \rightarrow \overline{\mathbf{C}}$ be a meromorphic function of degree $d \geq 2$ on a compact Riemann surface $X$ of genus $g$. Assume $\mathfrak{B}_{f} \leq$ $b(\delta)<\pi / 2$ where $b(\delta)$ is as in (3). Then $\delta<2$. We apply Proposition 8.4 and obtain a tiling of $X$ with a set $T$ of triangles. Let $S(\Delta)$ denote the 
sum of angles of the spherical triangle $\Delta^{\prime}=f(\Delta)$. Then by Lemma 7.2 we have $\left|\Delta^{\prime}\right|=S(\Delta)-\pi \leq \pi \delta$, which implies

$$
\left|\Delta^{\prime}\right| \leq \frac{\delta}{1+\delta} S(\Delta) \quad \text { for } \quad \Delta \in T .
$$

So we get, using (27), passing from summation over triangles to summation over vertices, and applying the Riemann-Hurwitz formula (18)

$$
\begin{aligned}
4 \pi d & =\sum_{\Delta \in T}\left|\Delta^{\prime}\right| \leq \frac{\delta}{1+\delta} \sum_{\Delta \in T} S(\Delta) \\
& =\frac{2 \pi \delta}{1+\delta} \sum_{p \in R_{f}} \mu_{f}(p) \leq \frac{4 \pi \delta}{1+\delta} \sum_{p \in R_{f}}\left(\mu_{f}(p)-1\right) \\
& =\frac{4 \pi \delta}{1+\delta}(2 d+2 g-2) .
\end{aligned}
$$

If $(g, d)=(0,2)$ or $(0,3)$, we get a contradition showing that $\mathfrak{B}_{f} \geq \pi / 2$.

If $(g, d) \neq(0,2),(0,3)$, we conclude $(1+\delta) / \delta \leq(2 d+2 g-2) / d$, which implies $\delta \geq d /(d+2 g-2)$. The claim follows.

Construction of Example 1.9.

Consider two equilateral spherical triangles $\Delta_{0}$ with angles $2 \pi / 5$ and $\Delta_{0}^{\prime}$ with angles $4 \pi / 5$. Let $f: \Delta_{0} \rightarrow \Delta_{0}^{\prime}$ be the homeomorphism conformal in the interior of $\Delta_{0}$, sending vertices to vertices. Let $\Sigma$ be the group of conformal and anticonformal automorphisms of the sphere, generated by reflections in the sides of $\Delta_{0}$. It is easy to verify directly that $\Delta_{0}$ is a fundamental region of this group, that is, $\left\{\gamma \Delta_{0}: \gamma \in \Sigma\right\}$ is a triangulation of the sphere with 20 equilateral triangles. The vertices of this triangulation correspond to the vertices of an inscribed icosahedron under an isometric embedding of the sphere into $\mathbf{R}^{3}$. By the Symmetry Principle $f$ extends to a continuous map $f: \overline{\mathbf{C}} \rightarrow \overline{\mathbf{C}}$, holomorphic except possibly at the vertices of the triangulation. Indeed, every vertex of our triangulation is common to 5 triangles. The image triangles, congruent to $\Delta_{0}^{\prime}$, of the triangles forming a cycle around a vertex have sum of angles $4 \pi$.

By the Removable Singularity theorem $f$ is a holomorphic map $f: \overline{\mathbf{C}} \rightarrow$ $\overline{\mathbf{C}}$. Hence it is a rational function. We have $\left|\Delta_{0}\right|=\pi / 5$ and $\left|\Delta_{0}^{\prime}\right|=7 \pi / 5$, so $\operatorname{deg} f=7$. All vertices of the triangulation are simple critical points, from which follows that $\mathfrak{B}_{f}$ is equal to the circumscribed radius of $\Delta_{0}^{\prime}$. 


\section{References}

[1] L. Ahlfors, Sur les fonctions inverses des fonctions méromorphes, C. R., 194 (1932), 1145-1147.

[2] W. Bergweiler and A. Eremenko, On the singularities of the inverse to a meromorphic function of finite order, Revista Mat. Iberoamericana, 11, 2 (1995), 355-373.

[3] A. Bloch, Les theéorèmes de M. Valiron sur les fonctions entières at la théorie de l'uniformisation, Ann. Fac. Sci. Univ. Toulouse (3), 17 (1926), $1-22$.

[4] R. Brody, Compact manifolds and hyperbolicity, Trans. Amer. Math. Soc., 235 (1978), 213-219.

[5] P. P. Belinskii, General properties of quasiconformal mappings, Nauka, Siberian division, 1974. (Russian)

[6] W. Chauvenet, A treatise on plane and spherical trigonometry, J. B. Lippincott Co, Philadelphia, 1850.

[7] H. Chen, Yosida functions and Picard values of integral functions and their derivatives, Bull. Austral. Math. Soc., 54 (1996), 373-381.

[8] J. Clunie, W. Hayman, The spherical derivative of integral and meromorphic functions, Comment. Math. Helv., 40 (1966), 117-148.

[9] M. de Guzman, Differentiation of integrals in $\mathbf{R}^{n}$, LNM 421, Springer, NY, 1975.

[10] W. Hayman, P. Kennedy, Subharmonic Functions, vol. 2, Academic Press, 1989.

[11] E. Hille, Ordinary Differential Equations in the Complex Domain, NY, Wiley, 1976.

[12] S. Lang, Introduction to Complex Hyperbolic Spaces, Springer, 1987.

[13] O. Lehto, K. Virtanen, Quasikonforme Abbildungen, Springer, 1965.

[14] A. Lohwater, Ch. Pommerenke, On normal meromorphic functions, Ann. Acad. Sci. Fenn., Ser AI, no. 550 (1973), 1-12. 
[15] D. Minda, Euclidean, hyperbolic and spherical Bloch constants, Bull. Amer. Math. Soc., 6 (1982), 441-444.

[16] D. Minda, Bloch constants for meromorphic functions, Math. Z., 181 (1982), 83-92.

[17] D. Minda, Yosida functions, Lectures on Complex Analysis (ed: Chuang Chi-Tai), Proc. Symp. Compl. Anal., World Sci., London 1988, 197-213.

[18] R. Nevanlinna, Analytic Functions, Springer, 1970.

[19] E. Peschl, Über unverzweigte konforme Abbildungen, Österreich. Akad. Wiss. Math.-Naturwiss. Kl. SB. II 185,(1976) 55-78.

[20] Ch. Pommerenke, Estimates for normal meromorphic functions, Ann. Acad. Sci. Fenn., Ser. AI, 476 (1970).

[21] G. Valiron, Recherches sur les théorème de M. Picard, Ann. Sci. École Norm. Sup., 38 (1921), 389-430.

[22] L. I Volkovyski, Converging sequences of Riemann surfaces, Mat. Sbornik 23, 3 (1948), 361-382 (Russian), Engl. transl.: AMS Translation Series, 2, vol. 32 .

[23] L. I. Volkovyskii, Researches on the type problem of a simply connected Riemann surface, Proceedings of Steklov Math. Inst., Academy of Sci. USSR, 34, 1950 (Russian).

[24] H. Wittich, Neuere Untersuchungen über eindeutige analytische Funktionen, Springer, Berlin, 1955.

[25] K. Yosida, On a class of meromorphic functions, Proc. Phys.-Math. Soc. Japan, 16 (1934), 227-235.

[26] L. Zalcman, A heuristic principle in function theory, Amer. Math. Monthly, 82 (1975), 813-817.

[27] L. Zalcman, Normal families: New perspectives, Bull. Amer. Math. Soc., 35, 3 (1998), 215-230.

Fachbereich Mathematik, MA 8-2, Technische Universität Berlin, Straße des 17. Juni 136, 10623 Berlin, Germany

bonk@math.tu-berlin.de

Purdue University, West Lafayette, Indiana 47907, U.S.A eremenko@math.purdue.edu 